\title{
BlackHoleCam: fundamental physics of the Galactic center ${ }^{*}$
}

Goddi C. ${ }^{1,2}$, Falcke H. ${ }^{1,3}$, Kramer M. ${ }^{3}$, Rezzolla L. ${ }^{4}$, Brinkerink C. ${ }^{1}$, Bronzwaer T. ${ }^{1}$, Davelaar J. R. J. ${ }^{1}$, Deane R. ${ }^{5}$, De Laurentis M. ${ }^{4}$, Desvignes G. ${ }^{3}$, Eatough R. P. ${ }^{3}$, Eisenhauer F. ${ }^{6}$, Fraga-Encinas R. ${ }^{1}$, Fromm C. M. ${ }^{4}$, Gillessen S. ${ }^{6}$, Grenzebach A. ${ }^{7}$, Issaoun S. ${ }^{1}$, Janßen M. ${ }^{1}$, Konoplya R. ${ }^{4}$, Krichbaum T. P. ${ }^{3}$, Laing R. ${ }^{8}$, Liu K. ${ }^{3}$, Lu R.-S. ${ }^{3}$, Mizuno Y. ${ }^{4}$, Moscibrodzka M. ${ }^{1}$, Müller C. ${ }^{1}$, Olivares H. ${ }^{4}$, Pfuhl O. ${ }^{6}$, Porth O. ${ }^{4}$, Roelofs F. ${ }^{1}$, Ros E. ${ }^{3}$, Schuster K. ${ }^{9}$, Tilanus R. ${ }^{1,2}$,

Torne P. ${ }^{3}$, van Bemmel I. ${ }^{10}$, van Langevelde H. J. ${ }^{10}$, Wex N. ${ }^{3}$, Younsi Z. ${ }^{4}$, Zhidenko A. ${ }^{4}$

${ }^{1}$ Department of Astrophysics/IMAPP, Radboud University, 6500 GL Nijmegen, the Netherlands

${ }^{2}$ ALLEGRO/Leiden Observatory, PO Box 9513, NL-2300 RA Leiden, the Netherlands

${ }^{3}$ Max-Planck-Institut für Radioastronomie, Auf dem Hügel 69, D-53121 Bonn, Germany

${ }^{4}$ Institut für Theoretische Physik, Goethe-Univ., Max-von-Laue-Str. 1, 60438 Frankfurt, Germany

${ }^{5}$ RATT, Department of Physics, Rhodes University, Grahamstown 6140, South Africa

${ }^{6}$ Max-Planck-Institut für extraterrestrische Physik, Garching bei München, Germany ${ }^{7}$ ZARM, University of Bremen, Am Fallturm, D-28359 Bremen, Germany

${ }^{8}$ ESO, Karl-Schwarzschild-Strasse 2, D-85748 Garching bei München, Germany ${ }^{9}$ IRAM, 300 rue de la Piscine, 38406 St. Martin d'Hres, France

${ }^{10}$ Joint Institute for VLBI in Europe, Postbox 2, 7990 A A, Dwingeloo, The Netherlands

Einstein's General Theory of Relativity (GR) successfully describes gravity. Although GR has been accurately tested in weak gravitational fields, it remains largely untested in the general strong field cases. One of the most fundamental predictions of GR is the existence of black holes (BH). After the recent direct detection of gravitational waves by LIGO, there is now near conclusive evidence for the existence of stellar-mass BHs. In spite of this exciting discovery, there is not yet direct evidence of the existence of BHs using astronomical observations in the electromagnetic spectrum. Are BHs observable astrophysical objects? Does GR hold in its most extreme limit or are alternatives needed? The prime target to address these fundamental questions is in the center of our own Milky Way, which hosts the closest and best-constrained supermassive $\mathrm{BH}$ candidate in the Universe, Sagittarius $A^{*}\left(\mathrm{Sgr} \mathrm{A}^{*}\right)$. Three different types of experiments hold the promise to test GR in a strong-field regime using observations of Sgr A* with newgeneration instruments. The first experiment consists of making a standard astronomical image of the synchrotron emission from the relativistic plasma accreting onto Sgr A*. This emission forms a "shadow" around the event horizon cast against the background, whose predicted size $(\sim 50 \mu$ as) can now be resolved by upcoming very long baseline radio interferometry experiments at $\mathrm{mm}$-waves such as the Event Horizon Telescope (EHT). The second experiment aims to monitor stars orbiting Sgr A* with the nextgeneration near-infrared interferometer GRAVITY at the Very Large Telescope (VLT). The third experiment aims to detect and study a radio pulsar in tight orbit about Sgr A* using radio telescopes (including the Atacama Large Millimeter Array or ALMA). The BlackHoleCam project exploits the synergy between these three different techniques and contributes directly to them at different levels. These efforts will eventually enable us to measure fundamental $\mathrm{BH}$ parameters (mass, spin, and quadrupole moment) with sufficiently high precision to provide fundamental tests of GR (e.g., testing the no-hair theorem) and probe the spacetime around a $\mathrm{BH}$ in any metric theory of gravity. Here, we review our current knowledge of the physical properties of Sgr A* as well as the current status of such experimental efforts towards imaging the event horizon, measuring stellar orbits, and timing pulsars around Sgr A*. We conclude that the Galactic center provides a unique fundamental-physics laboratory for experimental tests of $\mathrm{BH}$ accretion and theories of gravity in their most extreme limits. 


\section{Gravity, General Relativity and black holes}

Gravity governs the structure and evolution of the entire Universe, and it is successfully described by Einstein's General Theory of Relativity (GR). In fact, the predictions of GR have been extremely well tested in the "local" universe, both in the weak field limit (as in the Solar System an and for strongly self-gravitating bodies in pulsar binary systems. ${ }^{107}$ Nevertheless, gravity in its GR description remains the least understood of all forces, e.g., resisting unification with quantum physics. In fact, GR assumes a classical description of matter that completely fails at the subatomic scales which govern the early Universe. Therefore, despite the fact that GR represents the most successful theory of gravity to date, it is expected to break down at the smallest scales. Alternative theories have been considered in order to encompass GR shortcomings by adopting a semi-classical scheme where GR and its positive results can be preserved. ${ }^{32}$ So, does GR hold in its most extreme limit? Or are alternative theories of gravity required to describe the observable Universe? These questions are at the heart of our understanding of modern physics.

The largest deviations from GR are expected in the strongest gravitational fields around black holes (BHs), where different theories of gravity make significantly different predictions. The recent detection of gravitational waves ${ }^{1]}$ seems to indicate that even events associated with very strong gravitational fields, such as the merger of two stellar-mass BHs, fulfil the predictions of GR. This extremely exciting discovery calls for additional verification using observations in the electromagnetic spectrum. In fact, astronomical observations and gravitational wave detectors may soon provide us with the opportunity to study BHs in detail, and to probe GR in the dynamical, non-linear and strong-field regime, where tests are currently lacking.

Although BHs are one of the most fundamental and striking predictions of GR, and their existence is widely accepted, with many convincing BH candidates in the Universe, they remain one of the least tested concepts in GR: for instance, there is currently neither a direct evidence for the existence of an event horizon nor tests of BH physics in GR (e.g. "no-hair" theorem). So, are BHs just a mathematical concept, or are they real, observable astrophysical objects?

In order to conduct tests of GR using BHs as astrophysical targets, it is crucial to resolve with observations the gravitational sphere of influence of the $\mathrm{BH}$, down to scales comparable to its event horizon. The characteristic size scale of a $\mathrm{BH}$ is set by its event horizon in the non-spinning case, the Schwarzschild radius: $R_{\mathrm{Sch}}=2 R_{\mathrm{g}}=2 G M_{\mathrm{BH}} / c^{2}$, where $R_{g}$ is the gravitational radius, $M_{\mathrm{BH}}$ is the $\mathrm{BH}$ mass, $G$ is the gravitational constant, $c$ is the speed of light. The angular size subtended by the Schwarzschild radius for a $\mathrm{BH}$ at distance $D$ is: $\theta_{\mathrm{Sch}}=R_{\mathrm{Sch}} / D \approx 0.02$ nanoarcsec $\left(M_{\mathrm{BH}} / M_{\odot}\right)(\mathrm{kpc} / D)$. For stellar-mass BHs (with $\left.\sim 10 M_{\odot}\right), \theta_{\text {Sch }}$ lies obviously well below the resolving power of any current telescope. Supermassive black holes (SMBHs), which supposedly lie at the center of

aThe first test of GR was the Eddington's solar eclipse expedition of 1919.51 
most galaxies, are several orders of magnitude larger, but they are at correspondingly much larger distances, resulting in their angular size to be generally too small to be resolved by any observing technique. But there is a notable exception: the center of our own Galaxy, which hosts the closest and best constrained candidate $\mathrm{SMBH}$ in the Universe. This SMBH is a factor of a million larger than any stellarmass $\mathrm{BH}$ in the Galaxy and at least thousand times closer than any other SMBH in external galaxies, making it the largest $\mathrm{BH}$ on the sky and, therefore, a prime target for $\mathrm{BH}$ astrophysical studies and GR tests.

In this review, we first summarise the observed physical properties of the SMBH candidate in the Galactic center (\$2). We then describe current experimental and theoretical efforts of the BlackHoleCam ${ }^{\mathrm{b}}$ project, which is funded by the European Research Council (ERC) and is a partner of the Event Horizon Telescop $£^{\mathrm{C}}$ (EHT) consortium. Its main goals are to image the immediate surroundings of an event horizon as well as to understand the spacetime around a SMBH (both in GR and in alternative theories of gravity) using stellar and pulsar orbits as probes (\$3). We later argue that the combination of independent results from different experiments can lead to a quantitative and precise test of the validity of GR ( $\$ 3.4$ ) and effectively turn our Galactic center into a cosmic laboratory for fundamental physics, enabling gravity to be studied in its most extreme limit (44). For detailed reviews of tests of GR in the Galactic center, we refer to Refs. $90 \mid 91$

\section{The supermassive black hole in the Galactic center}

\subsection{Observational properties}

The astronomical source suspected to be the SMBH at the center of the Galaxy was first detected in the radio as a point source named Sagittarius $A^{*}\left(\operatorname{Sgr} A^{*}\right), 11$ and has subsequently been studied across the full electromagnetic spectrum. What makes Sgr A* unique is its close proximity, only about $8 \mathrm{kpc}, \frac{147}{14}$ along with its large mass, about $4 \times 10^{6} M_{\odot} \cdot \frac{75 \mid 76}{}$ Consequently, the physical properties of Sgr A* can be uniquely determined with a level of confidence not possible with other SMBH candidates, making it the most compelling case for the existence of a SMBH. Here we summarise its main observational parameters: mass (2.1.1, spectrum (\$2.1.2, size $(2.1 .3$, and accretion rate (\$2.1.4). For full reviews, see Refs. 70|72]126.

\subsubsection{Mass}

The best evidence for a central dark mass of a few million solar masses comes from near-infrared (NIR) studies with ground-based 8-m class telescopes, where the development of adaptive optics has provided the ability to track the motions of individual stars orbiting around Sgr A* over several decades. ${ }^{7174} 76$ So far, about 30 stellar orbits have been monitored in the center of our Galaxy ${ }^{\sqrt{75176}}$ (Figure 1 .

\footnotetext{
b http://www.blackholecam.org/.

${ }^{\mathrm{c}}$ http://www.eventhorizontelescope.org/.
} 
left panel). One of these stars (S2), with an orbital period of about 16 years and an orbital speed of about $10000 \mathrm{~km} \mathrm{~s}^{-1}$, has been followed for over one fullyclosed orbit around the $\mathrm{SMBH},{ }^{75 / 77}$ showing a textbook-like Keplerian elliptical orbit (Figure 1. middle and right panels). These measurements have provided a unique opportunity to map out the gravitational potential around Sgr A* with high precision, $\frac{76 / 129 / 152}{1}$ and demonstrated that this potential, in the central tenth of a parsec of the Milky Way, must be dominated by a single point source of a few million solar masses. ${ }^{[7576}$ The most precise measurement of the mass is yielded through combining measurements of stars orbiting about Sgr A*76 and in the old Galactic nuclear star cluster: ${ }^{34} M_{\mathrm{BH}}=4.23( \pm 0.14) \times 10^{6} M_{\odot}$ (see Ref. [34).
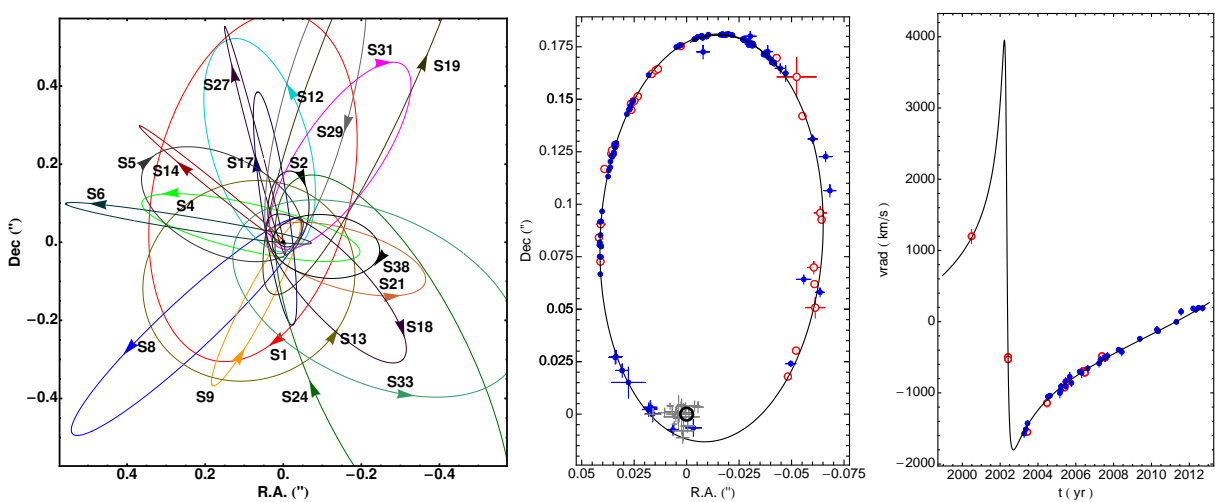

Fig. 1. (Left panel) Stellar orbits in the central arcsecond from Sgr A* (at the origin). (Middle and Right panels) Measured locations and radial velocity of the star S2 around Sgr A* (with the fitted orbit shown in black), measured with the NTT and the VLT (blue circles), and Keck (red circles) from 1992 until 2012.78 The radio position of Sgr A* is marked by a black circle and those of NIR flares from Sgr A* by grey crosses. Adapted from Ref. 78

The final piece of evidence needed to associate the measured dark mass with Sgr $A^{*}$ is provided by its own peculiar motion, which is consistent with $0(<0.4 \pm$ $0.9 \mathrm{~km} \mathrm{~s}^{-1}$ ), as measured with very long baseline interferometry (VLBI) using radio telescopes. $\frac{146}{16}$ When compared with the high velocities of the orbiting stars in the same region (up to $10^{4} \mathrm{~km} / \mathrm{s}$ ), the implication is that at least $10 \%$, if not all, of the dark mass must be associated with Sgr A* .146

The distance to Sgr $\mathrm{A}^{*}$ has also been accurately measured using both $3 \mathrm{D}$ velocities of orbiting stars measured with NIR telescopes $(D=8.33 \pm 0.11 \mathrm{kpc})^{34 / 75 / 76}$ and VLBI parallax measurements of molecular masers $(D=8.35 \pm 0.15 \mathrm{kpc}) .147$

Put together, these measurements have provided the clearest evidence for the existence of a SMBH at the center of our own Galaxy, and of BHs in general.

\subsection{2. (Radio) spectrum}

Despite the definition of a "black" hole, there is nonetheless some information reaching us from near the event horizon in the form of electromagnetic radiation. In- 
deed, gas and plasma around BHs are transported inwards through an accretion flow, which heats up the material and emits large amounts of energy. This energy is radiated across the entire electromagnetic spectrum from the radio, to infrared, optical, X-ray, and gamma-ray bands. Since optical radiation from the Galactic center is completely absorbed, the only observing bands where Sgr A* is clearly detected are the radio (including sub-mm waves), the NIR and mid-infrared (MIR), and X-rays (e.g., see Figure 2 in Ref. 57 for a broad-band spectrum of Sgr A*).

Combining all radio data, one finds that the radio flux density $S_{\nu}$ increases slowly with frequency $\left(S_{\nu} \propto \nu^{\alpha}\right.$ and $\left.\alpha \sim 0.3\right)$ and peaks at about $10^{3} \mathrm{GHz}(0.3 \mathrm{~mm}) . \underline{57}$ Observing this synchrotron emission at sub-mm waves rather than at longer wavelengths brings a two-fold advantage: the emission becomes optically-thin and comes from smaller scales (a typical property for self-absorbed synchrotron sources). Ref. 53 were the first to realize that such a "sub-mm bump" in the spectrum of Sgr A* implies a scale of the order of several $R_{\text {Sch }}$ in diameter, and used this argument to suggest that the event horizon of Sgr A* could be imaged against the background of this synchrotron emission using VLBI at (sub-)mm waves (see \$3.1.2).

\subsubsection{Size and structure}

Determining the intrinsic size and structure of Sgr A* from direct imaging is difficult, and not only because of its small size. In fact, scattering of radio waves by electrons in the interstellar medium (ISM), between us and the Galactic center, washes out any structure at long radio wavelengths, $\frac{160}{16 l u r r i n g}$ Sgr A* into an east-west ellipse of axial ratio 2:1.18119 The observed scatter-broadened angular size of Sgr A* follows a $\lambda^{2}$ law ${ }^{545}$ (see Figure 2, left panel): $\phi_{\text {scatt }}=(1.36 \pm 0.02) \operatorname{mas} \times(\lambda / \mathrm{cm})^{2}$.

Using a closure amplitude analysid def. 18 showed that the measured sizes of Sgr A* at $1.3 \mathrm{~cm}(22 \mathrm{GHz})$ and $7 \mathrm{~mm}(43 \mathrm{GHz})$ actually deviate from the predicted $\lambda^{2}$ law, owing to the contribution of the intrinsic size, which seems to decrease with frequency. Since the scattering effect reduces with increasing frequency, measurements at higher frequencies can more easily reveal such an intrinsic size. For instance, recently Ref. 139 measured an intrinsic 2D source size of $(147 \pm 7) \mu$ as $\times$ $(120 \pm 12) \mu \mathrm{as}$, at $3.5 \mathrm{~mm}(85 \mathrm{GHz})$. Fitting data acquired up to $230 \mathrm{GHz}$, Ref. 54 report an intrinsic size of $\phi_{\mathrm{Sgr} \mathrm{A}^{*}}=(0.52 \pm 0.03) \operatorname{mas} \times(\lambda / \mathrm{cm})^{1.3 \pm 0.1}$. At the wavelength of $1.3 \mathrm{~mm}(230 \mathrm{GHz})$, the angular size is $37 \mu$ as (Figure 2 , right panel), which although very small, is within reach of the VLBI technique (see \$3.1.2).

\subsubsection{Accretion rate}

After the mass, the most important parameter of an astrophysical BH is its accretion rate, since it determines the level of activity. The best estimates of the accretion

\footnotetext{
${ }^{\mathrm{d}}$ In radio interferometry, closure amplitudes are quantities formed by combining the complex amplitudes in the correlated "visibilities" measured between sets of four different telescopes such that telescope-based gain errors cancel out.157
} 

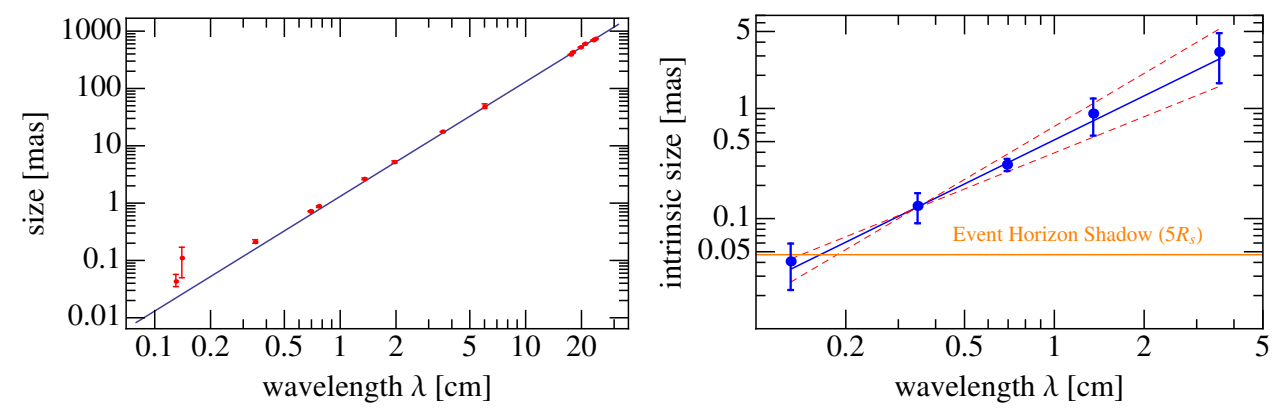

Fig. 2. (Left panel) Observed major-axis size of Sgr A* as a function of wavelength measured by various VLBI experiments. This size follows a $\lambda^{2}$ scattering law (indicated by the solid line). Size measurements on this line are dominated by scattering effects, while measurements falling above the line indicate intrinsic structure larger than the scattering size. (Right panel) Intrinsic size of Sgr A* derived after subtraction of the scattering law (see Ref. 54 for details). The systematic uncertainties in the scattering law are plotted as dashed red lines. The predicted event horizon size (3.1.1 is indicated with an orange line. Taken from Ref. 57.

rate onto Sgr $\mathrm{A}^{*}$ are provided by radio polarization measurements. In fact, the synchrotron radiation is typically linearly polarized, but the polarization vector rotates as the radio waves propagate through the magnetized ISM, an effect called Faraday Rotation, which has a simple dependence on the wavelength: $\Delta \phi=\mathrm{RM} \times$ $\lambda^{2}$, where $\mathrm{RM}=8 \times 10^{5} \mathrm{rad} \mathrm{m}^{-2} \int B(s) n_{e}(s) \mathrm{d} s$ is the rotation measure (RM) which represents the overall strength of the effect, $B$ is the line-of-sight magnetic field (in $\mathrm{G}$ ), $n_{e}$ is the thermal electron density (in $\mathrm{cm}^{-3}$ ), and $s$ is the path length (in pc) along the line-of-sight through the medium. $\frac{122}{12}$ The detection of strong linear polarization at (sub-)mm wavelengths ${ }^{23}$ provided a rotation measure of $R M \simeq$ $-6 \times 10^{5} \mathrm{rad} \mathrm{m}^{-2}, 122123$ the highest value ever measured in any astronomical source. Adopting this value and assuming a range of plausible density and magnetic field profiles, the accretion rate can be constrained to vary in the range $10^{-9} M_{\odot} / \mathrm{yr} \leq$ $\dot{M} \leq 10^{-7} M_{\odot} /$ yr on scales of hundreds to thousands of $\mathrm{R}_{\mathrm{Sch}} \cdot \frac{122 \mid 153}{10}$

\subsubsection{Puzzling aspects}

There are a few puzzling aspects regarding the physical properties of Sgr A* inferred from observations. Firstly, the estimated value for the accretion rate is at least four orders of magnitude below the average accretion rate required to grow a four million solar mass BH in a Hubble time. Secondly, the radio luminosity of Sgr A* is well below the typical values observed in low-luminosity Active Galactic Nuclei ${ }^{133}$ (AGN), indicating a remarkably low state in the activity level with respect to other SMBHs in galaxies. Thirdly, the amount of gas available for accretion around the $\mathrm{BH}$ would imply emission many orders of magnitude larger than observed (e.g., compare $\sim 10 \% \dot{M}_{\text {Bondi }} c^{2}=6 \times 10^{41} \mathrm{erg} / \mathrm{sec}$ to $\nu L_{\nu}(350 \mathrm{GHz}) \sim 10^{35} \mathrm{erg} / \mathrm{sec}$; see Ref. [57). This extremely low level of activity has led to competing models to explain the appearance of the emission from Sgr A*, which we discuss in next section. 


\subsection{Astrophysical models}

Since Sgr A* is the closest SMBH candidate, it is a natural testbed for accretion theories in AGN. Despite being the best-studied object of its kind, the exact nature of its emission processes, dynamics, and geometry are still rather uncertain.

As already pointed out, Sgr A* is highly underluminous, with a bolometric luminosity of $10^{-8}$ times the Eddington limit, which renders it an extreme case among the known population of AGN. In this regime, the emission is conventionally modelled as arising from a radiatively inefficient accretion flow (RIAF). $135|136| 172] \mathrm{In}$ such a model, the disk radiates inefficiently owing to low particle density which leads to a decoupling of electron and proton temperatures. $\frac{120}{120}$ The protons carry most of the mass (i.e. of the energy), whereas the electrons produce most of the radiation (via synchrotron, bremsstrahlung and inverse-Compton processes). Owing to this decoupling, most of the gravitational energy is viscously converted into thermal energy of the protons (which cool inefficiently), and only a small fraction of the dissipated energy is transferred to the electrons via Coulomb collisions and can be radiated away. ${ }^{173}$ Since unlike for the electrons the radiative cooling is inefficient for protons, most of the gravitational energy released by viscous dissipation (not radiated away by the electrons) is advected by the accreting gas and swallowed by the $\mathrm{BH}$, and one speaks of advection-dominated accretion flows (ADAF) 1351136

Besides RIAF, alternative mechanisms to reduce the radiative efficiency have been proposed. An interesting possibility is the reduction of the accretion rate via outflows. In the tradition of the ADAF models, $\frac{135 \mid 136}{2}$ Ref. 16 proposed the adiabatic inflow-outflow solution (ADIOS) model where the inflow/outflow rates decrease inward with decreasing radius according to $\dot{M}(r) \propto r^{p}$, where $0 \leq p<1$. Current dynamical models of the region near the Bondi radius $\frac{\sqrt{175 \mid 176}}{}$ are consistent with values of the outflow index of $p \sim 0.5-0.6$, showing the importance of outflows in the dynamics of the Galactic center. Spectral modeling from radio to X-ray

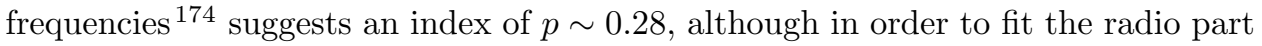
of the spectrum by either the RIAF or the ADAF models, an additional contribution of hot electrons $\left(\sim 10^{11} \mathrm{~K}\right)$ is required ${ }^{174}$ This population is often assumed to be due to a jet emitted from the very inner parts of the accretion flow. $56 \mid 1211131$

The current state-of-the art dynamical models of $\mathrm{BH}$ accretion are based on general relativistic magneto-hydrodynamic (GRMHD) simulations ${ }^{431124}$ that are typically initialized from a stationary rotating torus. $\frac{6465}{6 f}$ If the torus contains a weak magnetic field, the magnetorotational instability (MRI) ${ }^{12}$ arises, which leads to selfconsistent transport of angular momentum and mass accompanied by intermittent and unsteady outflows. $\frac{4311241125}{}$ In the presence of strong magnetic fields, a massive supply of ordered vertical magnetic flux builds-up near the $\mathrm{BH}$ until reaching saturation; as a consequence, the MRI is marginally suppressed and the accreting material enters the so-called magnetically arrested disk (MAD) state.125|156

To determine whether accretion and outflows in the Galactic center are in the regime of RIAF, ADIOS, MAD or something else entirely, GRMHD simulations 

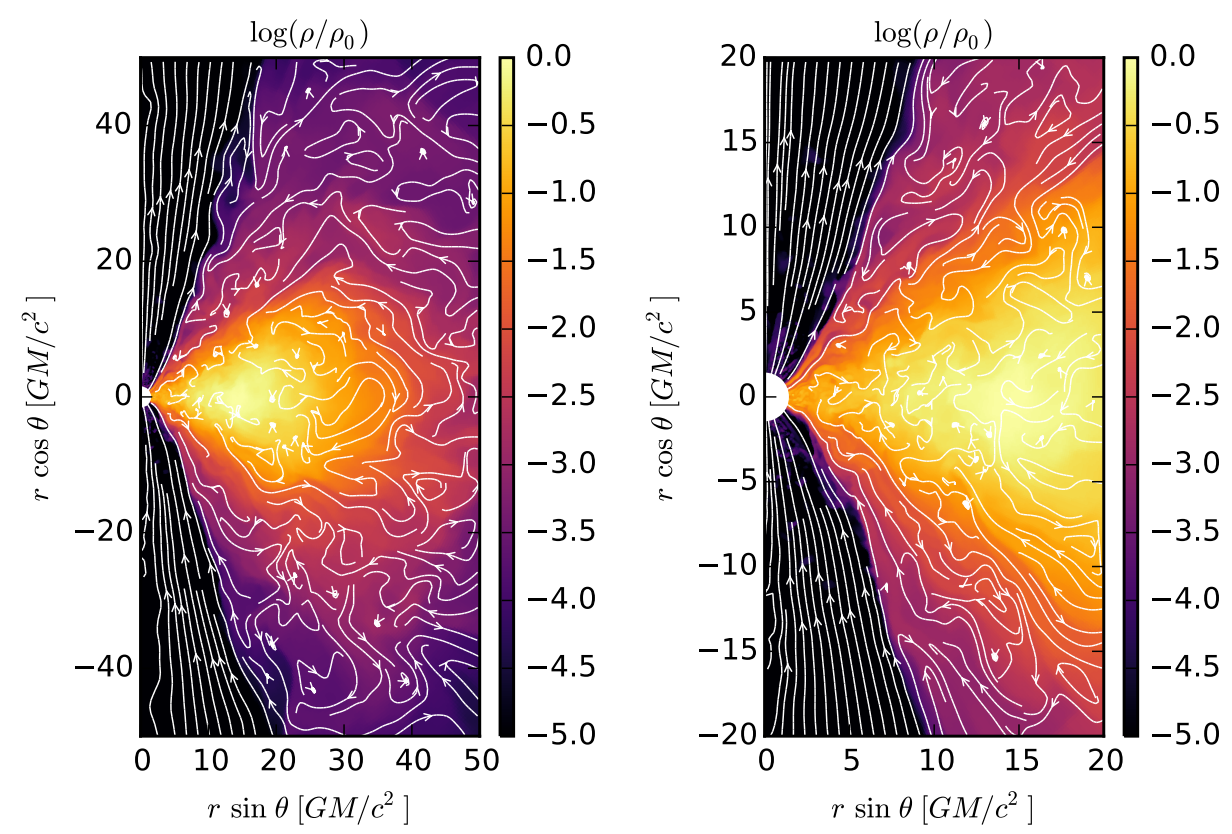

Fig. 3. GRMHD simulations performed with the BHAC code from Ref. 143, showing an accreting torus and a relativistic jet in a $\mathrm{BH}$. Axes are units of $\mathrm{R}_{g}$. The color scale shows the (dimensionless) logarithmic rest-frame density ( $\rho_{0}$ is the maximum torus density). The magnetic field lines are shown in white. Horizon penetrating (modified Kerr-Schild) coordinates are used (the outer horizon is indicated by the white circle). The MRI leads to turbulence in the torus interior which drives accretion. A relativistic jet emerges in the low density "funnel" near the polar regions above the equatorial plane. The right panel shows a zoom on the central region.

coupled to radiation transport calculations are required. In order to study accretion and outflows in challenging regimes, e.g. incorporating large scales (preferentially up to the Bondi radius $\sim 10^{5} \mathrm{R}_{\mathrm{g}}$ ), tilted-disk accretion and non-equilibrium thermodynamics, the BlackHoleCam collaboration has developed a Black Hole Accretion Code (BHAC). ${ }^{143}$ The latter is a newly developed adaptive-mesh-refinement (AMR) multi-dimensional GRMHD code, which is built on the MPI-AMRVAC toolkit $\frac{1011142}{1}$ and can solve the GRMHD equations on any background metric, allowing a parametrized exploration of accretion in various spacetimes (see \$3.1.4). The main advantage of the AMR implementation used in BHAC over uniform grid cases has been recently demonstrated. $\frac{127}{1}$ Figure 3 shows a high-resolution $2 \mathrm{D}$ GRMHD simulation of accretion in a torus surrounding a Kerr BH (spin $a=0.9375)$ obtained with the BHAC code. $\frac{143}{14}$ The simulation shows typical features of BH accretion, including an inner jet composed of ordered magnetic field lines threading the $\mathrm{BH}$ ergosphere, a shear-layer between the jet and the slower disk wind, a disk/torus with a "turbulent" inner part driven by the MRI which leads to accretion.

Whether or not Sgr $\mathrm{A}^{*}$ drives a relativistic jet is an open question. The observed spectrum,,$\frac{56}{6}$ the frequency-dependent size,, 18 and the observed radio time lags 2454 can in principle be explained as a scaled-down version of a relativistic jet from an 

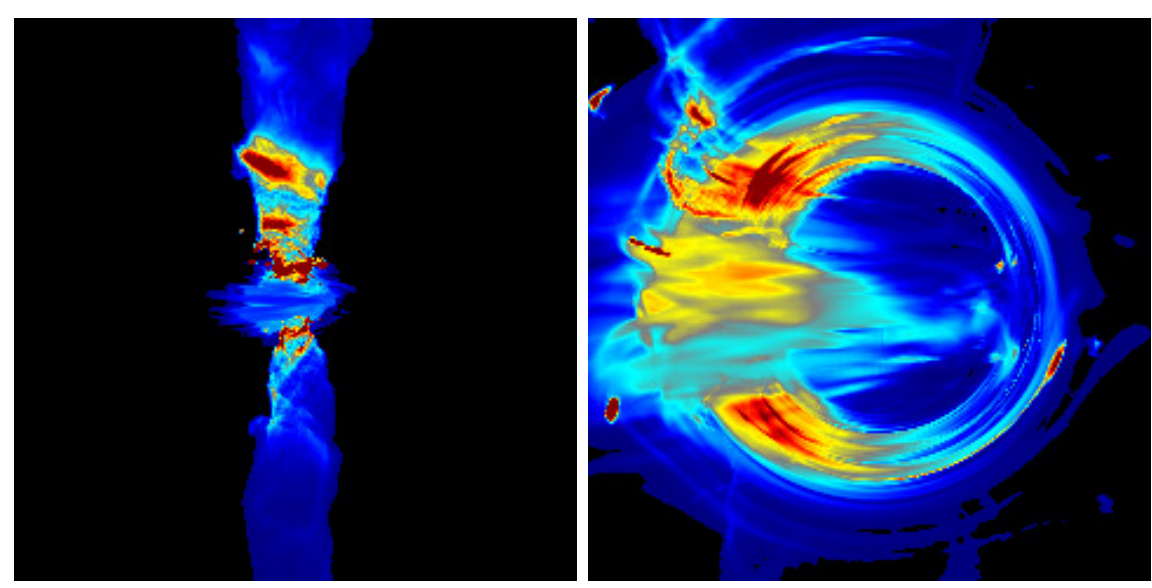

Fig. 4. Brightness distribution of the emission from relativistic jets produced in 3D-GRMHD simulations by Ref. 132 at $\lambda=7 \mathrm{~mm}$ (left panel) and $1.3 \mathrm{~mm}$ (right panel), respectively. Colors code the radiation intensity on a linear scale. A viewing angle $i=90^{\circ}$ is assumed. The fields of view are $200 \times 200 \mathrm{R}_{g}$ (left panel) and $20 \times 20 \mathrm{R}_{g}$ (right panel), respectively.

AGN but with very low accretion rate. ${ }^{55}$ In particular, 2D GRMHD simulations showed that jets can fully reproduce the flat-to-inverted radio-mm spectrum observed in $\operatorname{Sgr} \mathrm{A}^{*}, 1301131$ by requiring accretion rates of order of $10^{-9} M_{\odot} / \mathrm{yr}$ (i.e., at the lower end of the range estimated from radio polarization measurements; see 2.1.4. Interestingly, 3D GRMHD simulations predict the observational appearance of these relativistic jets at different frequencies ${ }^{132}$ (Fig. 4), which can in turn be directly compared with VLBI imaging experiments (see 3.1 .2 ).

It is worth noting that, since different models of Sgr $\mathrm{A}^{*}$ give different predictions for the appearance of the emission near the SMBH, this may impact our ability to discern strong gravity effects. Properly understanding the astrophysics is therefore crucial to investigate gravity on event horizon scales with astronomical techniques.

\section{Experimental tests of General Relativity and alternative theories of gravity within BlackHoleCam}

Based on the evidence summarized in $\$ 2$, we can now assess with great confidence that our Galactic center hosts the most compelling candidate SMBH in the Universe, and therefore naturally provides a prime target for astronomical observations which aim to assess the existence of BHs, test GR in the strong-field regime, and, more generally, study the spacetime around a $\mathrm{BH}$ (within GR and beyond).

In this section, we describe three different types of (on-going) experiments to test GR with astronomical observations of Sgr A*. The first experiment aims to study Sgr A* on horizon scales by imaging the relativistic plasma emission which surrounds the event horizon and forms a shadow cast against the background, which can be resolved using VLBI techniques at mm-wavelengths (\$3.1). The second experiment uses astrometric observations with NIR interferometry, which are expected 
to resolve orbital precessions of stars orbiting $\mathrm{Sgr} \mathrm{A}^{*}$ as well as hot spots on the innermost stable circular orbit (ISCO) around the SMBH, allowing measurements of the BH mass and spin ( $\$ 3.2)$. The third experiment relies on the detection and timing of radio pulsars in tight orbits around Sgr $\mathrm{A}^{*}$, which should reveal distinctive signatures in their orbits induced by the spin and quadrupole moment of Sgr A*, potentially providing the cleanest test of the no-hair theorem ${ }^{\mathrm{e}}(3.3)$. Although each type of observation may by itself lead to a measurement of the $\mathrm{BH}$ properties, it is effectively the cross-comparison of the predictions coming from different observational techniques that has the power to provide a fundamental test of GR (\$3.4). As argued later, ultimately, the results from all these measurements should be interpreted within a general theoretical framework for the $\mathrm{BH}$ spacetime, describing not only GR but also any possible alternative theory of gravity (3.1.4.

\subsection{Imaging the black hole shadow of Sgr A*}

\subsubsection{Definition of the shadow of a black hole}

The defining feature of a $\mathrm{BH}$ is the event horizon, the boundary within which a particle (or photon) cannot escape. As a consequence, BHs are completely black only within the event horizon, but outside $R_{\text {Sch }}$ light can escape. In fact the matter accreting onto the $\mathrm{BH}$ heats up via viscous dissipation and converts gravitational energy into radiation (2.2). So what would a BH actually look like, if one could observe it? Ref. 15] was the first to calculate the visual appearance of a $\mathrm{BH}$ against a bright background, and found that it is determined by a region of spherical photon orbits. Although the probability of a $\mathrm{BH}$ passing in front of a background source like a star is very small, Ref. 117 and later Ref. [58, building on the work of Ref. 15. showed that a $\mathrm{BH}$ embedded in an optically-thin emitting plasma (like the one expected to surround Sgr A*; see $\$ 2.1 .2$, would produce a specific observable signature: a bright photon ring with a dim "shadow" in its interior cast by the $\mathrm{BH}^{\mathrm{f}}$. The shadow is essentially an image of the photon sphere, lensed by the strong gravitational field around the $\mathrm{BH}$ and superimposed over the background light.

Owing to gravitational lensing, the size of the shadow is increased. In particular, compared to the angular radius of the $\mathrm{BH}$ horizon in a Euclidean spacetime $\left(R_{\mathrm{Sch}}=10 \mu\right.$ as at the distance of $\mathrm{Sgr} \mathrm{A}^{*}$ of $8.3 \mathrm{kpc}$; see $\$ 1$, relativistic calculations result in approximately a 2.5 times larger radius of the shadow. Therefore the angular diameter of the shadow in the sky is $\sim 50 \mu$ as as viewed from the Earthg, Although very small, this angular size can actually be resolved by VLBI at mm-

\footnotetext{
e The no-hair theorem $\frac{85 \mid 87 / 150}{}$ states that all (uncharged) BHs are uniquely described by only two parameters: the mass and the spin. This property is often referred to as "BHs have no hair". f Since photons orbiting around the BH slightly within the inner boundary of the photon region are captured by the event horizon while photons just outside of the outer boundary of the photon region escape to infinity, the shadow appears as a quite sharp edge between dark and bright regions. The first relativistic formula for the angular radius of a Schwarzschild BH was calculated by Ref. 155 Values for the angular diameter of the shadow of SMBHs are given in Refs. 80 81.
} 
wavelengths (see $\sqrt{3.1 .2}$ ), as first pointed out by Ref. [58.

In GR, the intrinsic size of the shadow $\left(\sim 5 R_{g}\right)$ is mainly determined by the $\mathrm{BH}$ mas $\xi^{\mathrm{h}}$, while its shape depends strongly on its spin and inclination. ${ }^{15529142}$ For a non-spinning, spherically-symmetric BH, the shape of the shadow is a perfect circle. For a Kerr BH, the difference in the photon capture radius between corotating and counter-rotating photons (with the corotating photons passing closer to the center of mass with increasing spin), creates a "dent" on one side of the shadow which depends on the $\mathrm{BH}$ spin. Moreover, the fact that photons passing on the counterrotating side have to pass at larger distances than the co-rotating side (to avoid being captured by the event horizon), results in the centroid of the shadow shifting significantly with respect to the mass center, resulting in crescent-like images. $\underline{44}$

Besides the geometrical shape, the emission brightness distribution also strongly depends on spin and inclination, with e.g. high-inclination, high-spin configurations having a more compact, one-sided structure (due to Doppler beaming) than lowspin, face-on configurations. Therefore, imaging the $\mathrm{BH}$ shadow can in principle enable one to constrain the spin and the orientation in the sky of the $\mathrm{BH}$.

In addition, sophisticated GRMHD models of the emission that include accretion disks and jets $44|121| 130-132]$ suggest that the observed emission morphology, besides GR beaming and lensing effects, depends also on the astrophysical model of the plasma flow. Therefore, the appearance of the shadow could also be used to discriminate between different models of the mm emission (e.g., disk vs. jet; see $\$ 2.2$.

Finally, if the no-hair theorem is violated, the shape of the shadow can become asymmetric $^{93}$ and its size may vary with parameters other than the BH mass, e.g. the $\mathrm{BH}$ quadrupole moment or generic parametric deviations from the Kerr metric. $\frac{315179 / 80188}{1 m a g i n g}$ the $\mathrm{BH}$ shadow can in principle provide constraints on these deviation parameters. Actually, since the shape of the shadow is set by the photon region, created by photons following (spherical) null geodesics in the spacetime around the BH, the morphology of the shadow is mainly determined by the theory of gravity assumed to govern the BH. Since the first study by Ref. [58, several groups have extended the calculations for the appearance of the BH shadow to a variety of spacetimes within GR and alternative theories of gravity (see 3.1.5. Therefore, $\mathrm{BH}$ shadow imaging experiments can test predictions for the properties of the shadow in alternative theories of gravity (see 3.1 .4 .

\subsubsection{Millimeter VLBI Imaging}

Radio interferometry is an astronomical observing technique to obtain highresolution images of radio sources. In particular, VLBI uses a global network of radio telescopes spread across different continents as an interferometer to form a virtually Earth-sized telescope. By recording radio wave signals at individual an-

${ }^{\mathrm{h}}$ The physical size has also a few \% dependence on the spin (see e.g., Ref. 92). The angular size will also be inversely proportional to the distance from the observer. 
tennas and afterwards cross-correlating the signals between all pairs of antennas post-facto (using time stamps of atomic clocks for synchronization), one obtains the so-called interferometric visibilities, that can be used to reconstruct an image of the source using Fourier transform algorithms. $\frac{157}{157}$ The achievable image resolution (in radians) of an interferometer is given by $\theta \sim \lambda / B$, where $\lambda$ is the observed wavelength and $B$ is the distance between the telescopes (or baseline). Hence, higher frequencies (shorter wavelengths) and longer baselines provide the highest resolving power. In fact, VLBI at mm wavelengths (mm-VLBI) offers the highest achievable angular resolution in ground-based astronomy, of the order of tens of microarcseconds ${ }^{i}$ which is sufficient to resolve the shadow cast by the BH in Sgr A* with an angular size on the sky of $\sim 50 \mu$ as (see 3.1 .1 ).

The first mm-VLBI observations of Sgr A* were conducted at $7 \mathrm{~mm}$ (or $43 \mathrm{GHz}$ ) using four stations of the Very Long Baseline Array. Although these provided evidence for source structure, they could not resolve the source with a synthesized beamsize of $\sim 2$ mas. $\frac{108}{10}$ Subsequent experiments carried out at $3 \mathrm{~mm}$ (or $90 \mathrm{GHz}$ ) started to resolve the source ${ }^{115}$ as well as to show evidence of asymmetric structure. $\frac{25[139}{}$ While observing at these relatively low frequencies is easier from a technical point of view (see below), there are three main scientific motivations for pushing VLBI observations of Sgr A* towards higher frequencies, or shorter wavelengths of about $1 \mathrm{~mm}$. First, the longest (i.e. Earth-sized) baselines can provide an angular resolution of $\sim 25 \mu$ as at $1.3 \mathrm{~mm}$, sufficient to resolve the shadow in Sgr A*. Second, the intrinsic size of the emission from $\mathrm{Sgr} \mathrm{A}^{*}$ is larger at longer wavelengths, $18|20| 115$ indicating that the observed emission is optically thick, obscuring the shadow near the $\mathrm{BH}$ for $\lambda \gtrsim 1 \mathrm{~mm}$. Third and most problematically, the blurring effect of the interstellar scattering dominates the size measurement at $\lambda>3 \mathrm{~mm}$, while at $1.3 \mathrm{~mm}$ a point source would be scattered to $\sim 22 \mu \mathrm{as}$, smaller (although still significant) with respect to the intrinsic source size (37 $\mu$ as; see $\$ 2.1 .3$ ).

While high frequencies are better suited to spatially resolving the BH shadow, mm-VLBI faces significant observational and technical challenges, i.e. higher data rates, higher stability required for atomic clocks and receiver chains, and, above all, the distortion effect of the wave fronts by the troposphere. Moreover, telescopes operating at mm-wavelengths are hard to build, because their surface accuracy needs to be much smaller than the wavelength they measure (i.e. $<<1 \mathrm{~mm}$ ). Building large dishes ( $>10 \mathrm{~m}$ in diameter) with such an accuracy is difficult. This explains why mm-VLBI experiments so far have been conducted with a limited number of stations (2-4), providing a minimal set of baselines which produce too few visibilities to form a high-fidelity image using the usual Fourier transform techniques. ${ }^{61} \mathrm{Nev}$ ertheless, although the current data are too sparse for imaging, one can in principle

\footnotetext{
iThe highest resolution ever obtained on the ground, yielding $\theta \sim 28 \mu$ as, was recently achieved at $1.3 \mathrm{~mm}$ (or $230 \mathrm{GHz}$ ) for a separation of $B \sim 9447 \mathrm{~km}$ between telescopes in Hawaii and Chile. 162 Using a space-based 10-m antenna, RadioAstron, a similar resolution was recently obtained also at longer radio wavelengths of $1.35 \mathrm{~cm} .97$
} 
use simulated images of the accretion flow to fit against the measured interferometric visibilities (an example is shown in Figure 5). This (non-imaging) approach has in fact already provided major breakthroughs (we provide a short summary below).

Ref. 109 were the first to detect Sgr A* at $1.4 \mathrm{~mm}(215 \mathrm{GHz})$ on a single baseline between the IRAM 30-m antenna at Pico Veleta in Spain and one 15-m antenna of the IRAM interferometer at Plateau de Bure in France $(1150 \mathrm{~km})$. After these first VLBI experiments with an Intra-European baseline,,$\frac{82}{}$ the subsequent experiments were conducted at a wavelength of $1.3 \mathrm{~mm}(230 \mathrm{GHz})$ with a three-station array (in Arizona, California, and Hawaii). The first remarkable result obtained with such an array is the discovery of resolved structure in Sgr $A^{*}$ on scales of only $4 \mathrm{R}_{\mathrm{Sch}}(\sim 40$ $\mu \mathrm{as})$, by measuring the correlated flux density as a function of projected baseline length. ${ }^{46}$ These initial measurements however did not allow an assessment of the exact nature of this structure or discrimination between Gaussian and ring models (the latter are motivated by the prediction of the shadow in front of the $\mathrm{BH}$ ). Besides measuring the source flux density at different baselines, which is sensitive to the source size, measurements of the closure phase $4^{\mathrm{j}}$ can provide some basic information about the orientation and the structure of the source, and turned out to be quite constraining in ruling out various models. For instance, Refs. $26 \mid 27$ argue that face-on models are highly disfavoured by current data, which seem instead to indicate that the disk spin axis is highly inclined to line of sight (but still exclude pure edge-on configurations). Ref. 63 have recently found that the median closure phase of Sgr $\mathrm{A}^{*}$ is nonzero, conclusively demonstrating that the $\mathrm{mm}$ emission is asymmetric on scales of a few $R_{s c h}$, as predicted by GR (see 3.1 .1 . In addition, Ref. 60 demonstrated that this small-scale emission from Sgr $A^{*}$ is also time variable, as expected in a relativistic accretion flow. Finally, Ref. 96 performed VLBI measurements of the linearly polarized emission and found evidence for (partially) ordered magnetic fields near the event horizon, on scales of $\sim 6 \mathrm{R}_{\mathrm{Sch}}$.

While this fitting technique in the Fourier domain has already been quite successful, providing major breakthroughs, spatially-resolved images on event-horizon scales are clearly necessary for assessing the nature of complex structure surrounding the shadow as well as for unambiguously determining $\mathrm{BH}$ properties such as its spin and inclination. To reach the goal of imaging the $\mathrm{BH}$ shadow, the crucial point is that the array should include more than three antennas and the resulting baselines should include both east-west and north-south orientations across different continents. For this purpose, an international collaboration, including BlackHoleCam, is assembling the Event Horizon Telescope (EHT), a mm-VLBI network of existing (and up-coming) mm-wavelength telescopes spread across several continents to form

${ }^{\mathrm{j} C l o s u r e-p h a s e s}$ are given by the sum of visibility phases along a closed triangle of stations in a VLBI array and they are very useful observables because they are robust against most phase corruptions induced by the atmosphere as well as the instrumentation.

${ }^{\mathrm{k}}$ Recent measurements of closure-phases at the longer wavelengths of $3 \mathrm{~mm}$ and $7 \mathrm{~mm}$, confirmed this result at larger radii.25|139|145 

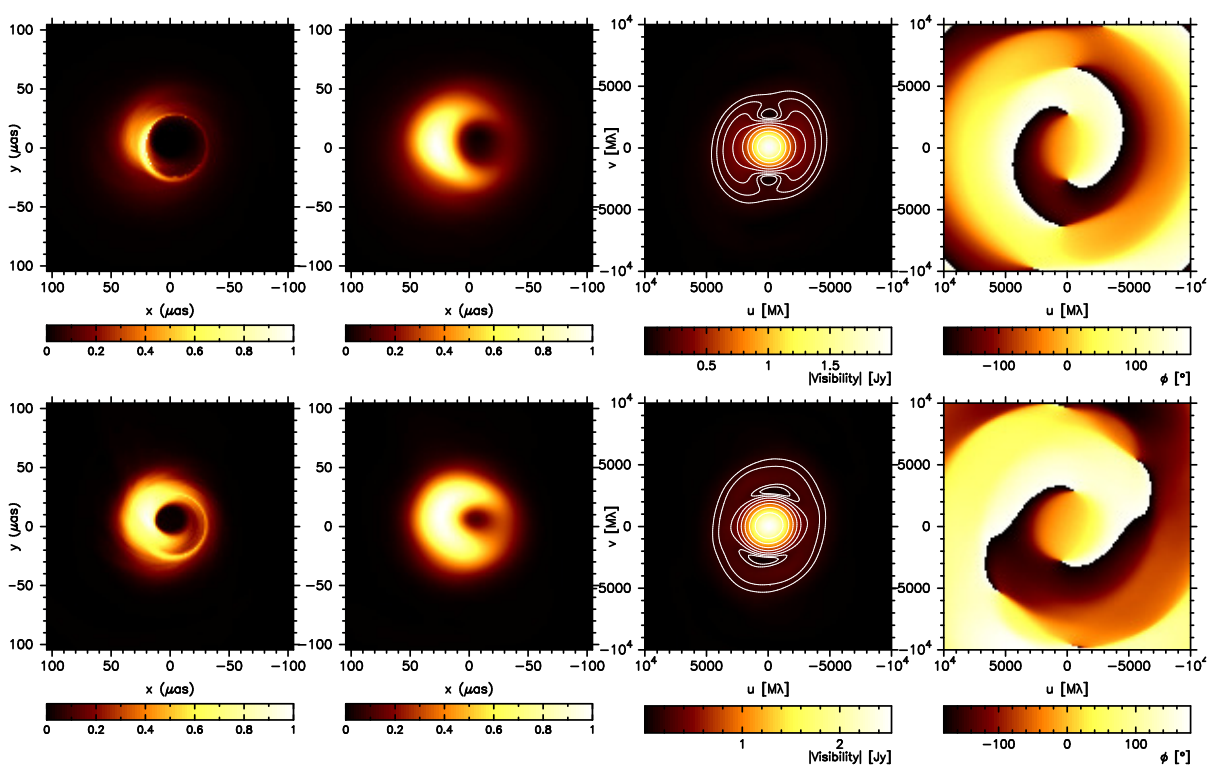

Fig. 5. Disk and jet models at $\lambda=1.3 \mathrm{~mm}$ from GRMHD simulations from Ref. 67, Left to right panels show an image of the disk (top row) and jet (bottom row) models, the same images convolved with the scattering screen, the visibility amplitudes, and the visibility phases of the scatter-broadened images (an inclination of $30^{\circ}$ is assumed). The color scale in the two left panels indicates the (normalized) radiation intensity. The shadow is clearly visible in both cases.

a global interferometer 1 Currently, the EHT operates at a wavelength of $1.3 \mathrm{~mm}$ $(\sim 230 \mathrm{GHz})$ and in the near future the VLBI capability may become available at higher frequencies $(\sim 350 \mathrm{GHz}) \cdot \frac{[158}{\square} \mathrm{A}$ critical element in the implementation of this plan is the Atacama Large Millimeter Array (ALMA), which is the most sensitive (sub)mm-wave telescope ever built and consists of 50 individual antennas of 12-m diameter. The inclusion of ALMA as a phased-array ${ }^{\mathrm{m}}$ will enable a transformative leap in capabilities, including unprecedented sensitivity and greatly improved image fidelity thanks to the north-south baseline. ${ }^{61}$ Joint VLBI observations that include ALMA as a phased array with other telescopes worldwide will start in 2017.

\subsubsection{Shadow measurement accuracy and interferometric simulations}

In order to use the interferometric image of the BH shadow to reveal potential deviations from the Kerr metric (see 3.1.4 and 3.1.5, we need to measure the fractional asymmetry of the shadow shape with respect to its angular size to the few percent

${ }^{1}$ The EHT includes mm-telescopes in Europe (IRAM Pico Veleta, and the up-coming phasedNOEMA), USA (JCMT/SMA, SMTO, KPNO), Mexico (LMT), South America (APEX, ALMA), and South Pole. For more details, please visit http://www.eventhorizontelescope.org

${ }^{\mathrm{m}} \mathrm{A}$ beamformer for ALMA has been developed that can aggregate the entrre collecting area of the array into a single, very large aperture (equivalent to an 84-m diameter telescope). In such a phased-array all antennas are combined to act jointly as a single dish that can operate as one giant element in a VLBI experiment. 
level. To achieve this goal, it is crucial to define the accuracy with which the BH shadow can be measured with the EHT. This requires a fundamental understanding of both the intrinsic properties of the source as well as the corruptions along the signal path, from the intervening ISM to correlator output. Furthermore, the efficacy of calibration and image reconstruction algorithms must be clearly understood and appropriately employed. All these components have both statistical and systematic uncertainties that need to be quantified to ensure a robust analysis.

Sources of uncertainties. An important source of uncertainty stems from the assumption that the intrinsic mm-wave sky brightness distribution of $\mathrm{Sgr} \mathrm{A}^{*}$ is not time-variable at sub-mas scales. In reality, variations in the accretion flow render the source variable on timescales comparable to the period of the ISCO, ranging from a few minutes (for a maximally rotating Kerr $\mathrm{BH}$ ) to about half an hour (for a Schwarzschild $\mathrm{BH}$ ). The challenge is that a source that is time-variable within the observation length breaks a simplifying assumption typically used for standard Earth-rotation aperture-synthesis imaging, upon which VLBI is based. ${ }^{157}$ Recent simulations of realistic EHT observations have nevertheless demonstrated that an image of the BH shadow can still be recovered by observing over multiple days and imaging the concatenated dataset, by effectively scaling the visibility amplitudes using the shortest baselines in the array. ${ }^{116}$ While this technique improves the image fidelity and dynamic range, it effectively averages out much of the information measured by the longest baselines as a trade-off. An interesting opportunity is that some of this variability may be dominated by a single blob of material accreting onto the $\mathrm{BH}$, and one could in principle track such a "hot spot" over many orbits within a single observing run, using it as a test particle to probe the Kerr spacetime using both closure quantities and/or direct imaging 29/47/59/95 (see also 3.2 .

In addition to intrinsic source variability, the refractive substructure of ISM inhomogeneities impose an apparent time variability (with a characteristic timescale of about one day). This is mitigated to a degree if data are collected over a period of time longer than the refractive timescale, resulting in what is known as the ensemble-average. ${ }^{134}$ This ensemble-average suffers from angular broadening due to the ISM, but the scattering properties are largely deterministic over most of the relevant range of baseline-lengths and wavelengths. As such, Ref. 62 have applied a reconstruction algorithm to a simulated EHT image that included scatterbroadening ${ }^{26}$ and demonstrated that the ISM blurring is invertible to a degree.

Another potential cause of uncertainty is the unknown structure of the accretion flow of Sgr $\mathrm{A}^{*}$ (see $\$ 2.2$. Although the accreting plasma could have density and magnetic field gradients both along and across the accretion disk, or even include a jet or a wind, we expect these uncertainties to play only a minor role, because the size and shape of the shadow are mainly determined by the spacetime (see 3.1 .5 .

The image reconstruction will finally be affected by statistical and systematic errors that stem from EHT data calibration, largely due to instrumental and atmospheric effects. In early VLBI observations with a three-station array, the (relative) 
amplitude calibration uncertainty was estimated to be around $5 \% . \frac{60}{6}$ For larger EHT arrays, one could use individual phased-interferometers (ALMA, SMA, NOEMA), which, besides the beam-formed data stream, may also simultaneously record local interferometric data at $\sim 0.01-1$ arcsec angular resolution. This enables calibration of the amplitude scale across the array under the assumption that the source flux is dominated by the sub-mas emission. Even more critical is the accurate calibration of the visibility phases, given that they carry the information on the spatial structure of the accretion flow. At $\mathrm{mm}$ wavelengths the effect of the troposphere on the visibility phases is significant, resulting in a "coherence" time that is typically 10 seconds at $\mathrm{mm}$ wavelengths and preventing the coherent time averaging on longer timescales. This ultimately limits the ability to perform highly accurate phase calibration due to the troposphere-induced signal-to-noise limit.

In order to gain a deep understanding of how these effects impact EHT observations and the robustness of any scientific inference that may result, it is clear that a detailed instrument simulator is required.

Tying measurements to theory: the need for realistic mm-VLBI simulators. As mentioned above, measuring the shape of the $\mathrm{BH}$ shadow at the few percent level requires prior knowledge, at a comparable level, of all the sources of uncertainty that affect the observations. In addition, radio interferometers, and in particular VLBI arrays which have relatively few individual stations, do not sample all spatial frequencies on the sky. Therefore, an image generated from an interferometric observation does not necessarily represent the full sky brightness distribution. Understanding all of the above effects to the required level of detail necessitates the simulation of the full signal path, quantifying all systematic contributions on the data products in particular (i.e. observed visibilities, closure quantities, reconstructed images). This instrument simulator can tie theoretical models to instrument measurements, by providing a framework to convert astrophysical model images/parameters (e.g. from GRMHD simulations) into simulated visibilities with realistic signal corruptions. The key point is to extract BH parameters, and therefore compare theoretical models directly from EHT visibilities.

For this purpose, in BlackHoleCam we are adopting the interferometry simulation software MEQTREES, $\stackrel{138}{ }$ initially developed for low-frequency interferometers (LOFAR and SKA). MEQTREES is a simulation and calibration package for building so-called "Measurement Equation Trees". 83 The visibilities measured by the interferometer are expressed using a chain of Jones matrices ${ }^{99}$ whose individual terms describe various independent instrumental and physical effects affecting the astronomical signal. The user can simulate any interferometric observation, by specifying the antenna configuration, observing frequency, instantaneous bandwidth, start time, etc. The individual Jones terms in the measurement equation then enable a simulation of the signal propagation and hence measured visibilities. Of course, if the effects can be simulated, then the process can be inverted and an arbitrary subset of the Jones matrix parameters can be solved for. 
Based on MeqTrees, a mm-VLBI specific software package called MeqSiLHouette, has been developed ${ }^{177}$ MeqSilhouette contains a series of components (or modules), including: a basic input module to convert theoretical model images into a sky model (which can be time-variable), a physically realistic approximation of both the mean and turbulent troposphere, a full treatment of time-variable ISM scattering, as well as time-variable antenna pointing errors (which are non-negligible relative to the station primary beams at $\mathrm{mm}$ wavelengths). In the future, additional effects can be included into the MeqSilhouette framework, as our understanding of the EHT increases over time. MeqSilhouette performs all steps in the Measurement Set data format ${ }^{\mathrm{m}}$. While it currently only performs total intensity simulations, its capability will be extended to full polarization in the near future.

One of the key points of this is to provide a realistic end-to-end simulator for the data calibration pipeline. For example, as input we can provide an emission model of a $\mathrm{BH}$ with a given spin, mass and position angle. MeqSilhouette then simulates an observation with the EHT with an arbitrary selection of realistic instrumental, ISM scattering and tropospheric effects. The resulting data are fed into the VLBI data processing pipeline, enabling an independent assessment of how well physical parameters of the $\mathrm{BH}$ input model are recovered, along with the statistical and systematic uncertainties. The next step is to use this end-to-end simulator, in which we can test the effect of a change in any given theoretical model parameter on the recorded visibilities. The end goal is to turn this simulator into a calibration pipeline and enable joint fitting of instrumental and scientific parameters. The motivation for this is to fully explore degeneracies between all parameters, scientific or calibration-related and so extract the maximum scientific inference from a given EHT dataset. This will of course employ standard Bayesian techniques.

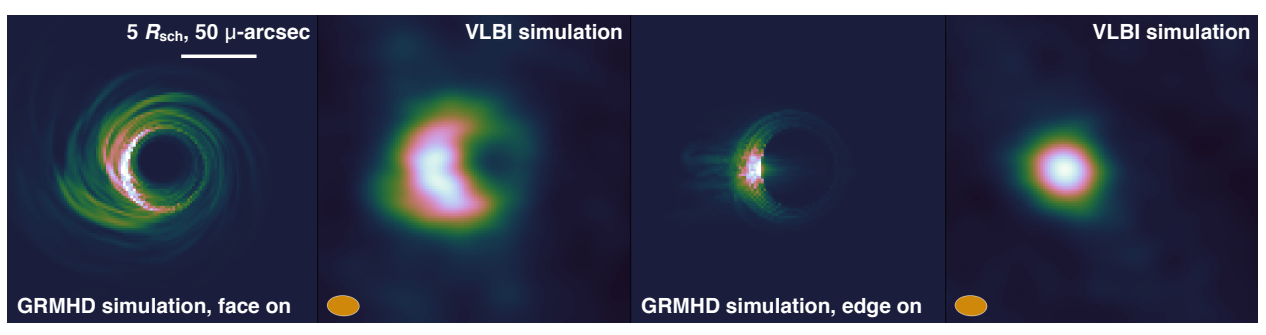

Fig. 6. GRMHD simulation 130 of the emission in an accretion flow around a rapidly spinning $\mathrm{BH}$ in Sgr $\mathrm{A}^{*}$. This is compared to a reconstructed image from simulated mm-VLBI data using MeqSilhouette, 17 for face-on and edge-on orientations of the accretion flow. The simulation assumes a 12 hour observation at $230 \mathrm{GHz}$, elevation limits of $15^{\circ}, 16 \mathrm{GHz}$ bandwidth, and implements the expected blurring from ISM scattering. The orange ellipse indicates the beam size.

Figure 6 shows an example of simulated images of the $\mathrm{BH}$ shadow generated with

\footnotetext{
${ }^{\mathrm{n}}$ The Measurement Set is a standard format for interferometric data, that describes the full observational setup and includes observational settings (metadata) such as station sensitivity, weather conditions, observing time and frequency, bandwidth, number of stations, etc.
} 
the MeqSilhouette software, $\frac{17}{17}$ for face-on and edge-on orientations of the accretion flow. The model is based on GRMHD simulations of Sgr A* by Ref. 130 and an EHT array that will be operational during the next few years (see 3.1 .2 . In the optimal case (face-on), the shadow is easily visible, while in the most pessimistic case (edge-on) a dynamic range $\geq 200: 1$ is needed to reveal the faint photon ring. This demonstrates the need for sophisticated imaging algorithms as well as robust Bayesian parameter estimation and model selection to achieve the scientific goals.

Expected accuracy. A number of theoretical studies have already started estimating the accuracy expected in EHT images. Ref. 149 utilized asymmetric crescents models to fit mock EHT data, and quoted an accuracy of about $1 \mu$ as. Ref. 89 used a simulated one-day observing EHT run with seven antennas, and demonstrated that the radius of the shadow of Sgr A* can be measured to an accuracy of $\sim 1.5 \mu$ as (corresponding to $6 \%$ ). Ref. 144 quoted an uncertainty of the same order $(\sim 0.9 \mu \mathrm{as})$, estimated using reasonable assumptions for the relative flux of the photon ring and the expected signal-to-noise achievable with the full EHT (extrapolated from the existing EHT observations). The MEQSilHouetTe end-toend simulator will build on this work and take the next step towards estimating the accuracy level to which the BH shadow can be recovered by the EHT.

\subsubsection{Black hole parameterization in general metric theories of gravity}

The absence of a quantum theory of gravity as part of a grand unified theory of all fundamental forces has resulted in the formulation of several alternative theories of gravity. In particular, we focus here on a class known as metric theories of gravity, where the spacetime has a symmetric metric, the trajectories of freely falling test bodies are geodesics of that metric, and in local freely falling reference frames, the non-gravitational laws of physics are those of special relativity. It is well known that such metric theories of gravity are built and classified according to the types of fields they contain, and the modes of interaction through those fields. Since they are strictly dependent of the field equation and because of the large number of alternative theories of gravity, including the possibility that the "true" theory is still unknown, it is reasonable to develop a model-independent framework which parametrises the most generic BH geometry through a finite number of adjustable quantities. These quantities must be chosen in such a way that they can be used to measure deviations from the general-relativistic BH geometry (Kerr metric) and could be estimated from the observational data. 161 This approach is similar in spirit to the parametrized post-Newtonian approach (PPN) which describes the spacetime far from the source of strong gravity. $\frac{165}{16}$ The main advantage of this approach is that different theories of gravity can be constrained at once?

One of the first such parameterisations for BHs was proposed by Ref. 94, who

\footnotetext{
${ }^{\circ}$ Given the large number of theories of gravity, a case-by-case validation of a given theory through cross-comparison with observations is obviously not an efficient approach.
} 
expressed deviations from GR in terms of a Taylor expansion in powers of $M / r$, where $M$ and $r$ are the $\mathrm{BH}$ mass and a generic radial coordinate, respectively. While some of the first coefficients of the expansion can be easily constrained in terms of PPN-like parameters, an infinite number remains to be determined from observations near the event horizon. ${ }^{94}$ As pointed out by Ref. 33 , this approach faces a number of difficulties, chiefly: i) the proposed metric is described by an infinite number of parameters which become roughly equally important in the strong-field regime; ii) the transformation from a spherically symmetric parameterization to an axially symmetric metric is performed through the Janis-Newman coordinate transformation, $\frac{137}{, 3 h i c h}$ is shown to be invalid in the general case. Therefore, the metric proposed by Ref. 94 cannot be used as a general and effective parameterization of an axially symmetric BH spacetime (see also Ref. 103 for more details).

A solution to these issues was proposed by Ref. 148 for arbitrary spherically symmetric, slow rotating BHs in metric theories of gravity. This was achieved by expressing the deviations from GR in terms of a continued-fraction expansion via a compactified radial coordinate defined between the event horizon and spatial infinity. The superior convergence properties of this expansion effectively permits one to approximate a number of coefficients necessary to describe spherically symmetric metrics to the precision that can be in principle achieved with near-future observations (see 3.1 .3 . Ref. 103 extended this new parametric framework by using a double expansion (in the polar and radial directions) to describe the spacetime of axisymmetric BHs in generic metric theories of gravity. This approach is phenomenologically effective, because it allows one to describe an arbitrary axiallysymmetric BH metric in terms of a relatively small number of parameters with a well-established hierarchy. Moreover, a number of well-known axially-symmetric metrics, such as Kerr, Kerr-Newman, higher dimensional Kerr projected on the brane ${ }^{100}$ and others, can be reproduced exactly throughout the whole spacetime with this parametrisation. The latter can also provide a convergent description for axially symmetric BHs in the Einstein-dilaton theory (Kerr-Sen $\mathrm{BH}^{[151}$ ) and in Einstein-Gauss-Bonnet-dilaton gravity. We expect therefore that such parametrised approach will be useful not only to study generic $\mathrm{BH}$ solutions, but also to interpret the results from mm-VLBI observations of the Sgr A* SMBH.

\subsubsection{Images of black hole shadows in generic spacetimes}

The primary science goal of BlackHoleCam is to capture and to study the image of the $\mathrm{BH}$ shadow in Sgr $\mathrm{A}^{*}$. Since its appearance depends on the assumed theory of gravity (3.1.1), its detailed shape provides an excellent observable test of GR and alternative theories of gravity. Indeed, several authors have calculated the appearance of a BH in known spacetimes, either within $\mathrm{GR}^{2[13|15| 42 \mid 79} \mid$ 81|140|170|177 or within alternative theories of gravity. ${ }^{4 / 5 / 7 / 8 / 14 \mid 171}$ Figure 7 shows several examples of shadows of Kerr and Kerr-like axisymmetric BHs.

An obvious problem that arises from using the detailed shape of the shadow to 

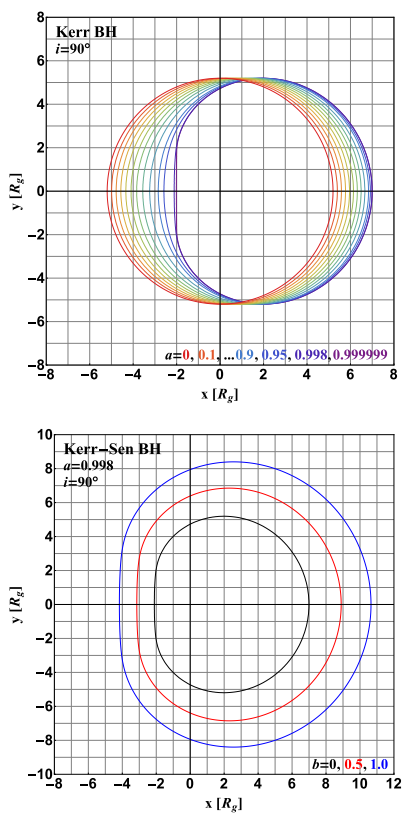
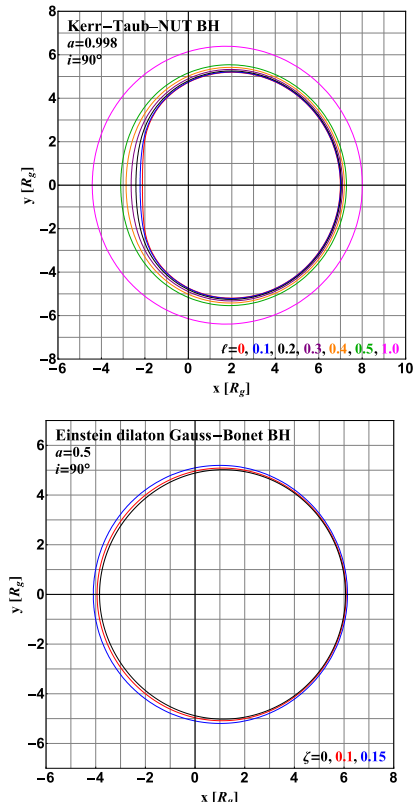
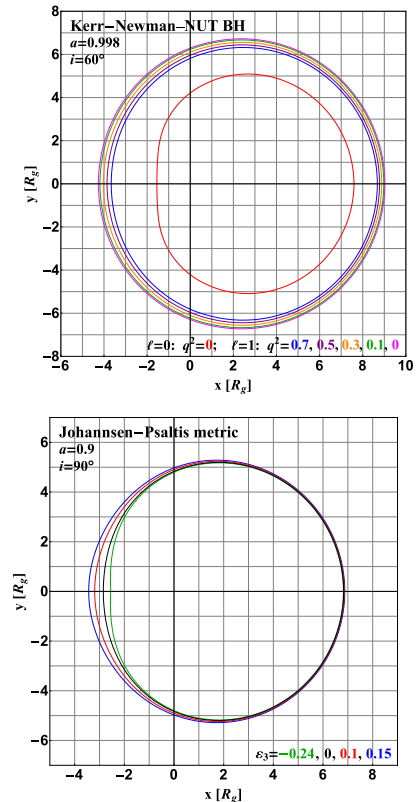

Fig. 7. Collection of BH shadow boundary curves. From left to right and top to bottom: Kerr $\mathrm{BH}$ with varying spin parameter (as reference), Kerr-Taub-NUT BH, Kerr-Newman-NUT BH, Kerr-Sen BH, Einstein dilaton Gauss-Bonet BH and Johannsen-Psaltis metric, 92 respectively. Adapted from Ref. 171 (panel 3 is from Ref. 79). In all panels the inclination angle $(i)$ is fixed as $90^{\circ}$, except for the third panel where it is $60^{\circ}$. The text in each panel details the specific BH spin and deformation parameters used in the shadow calculation.

test different theories of gravity, is its mathematical description. For example, in the case of a Kerr BH, the shadow is approximated as a circle, and then its deformation is measured by taking the ratio of the size of the dent to the radius of the circle. While this approach works well for Kerr BHs, it may not work equally well for $\mathrm{BH}$ spacetimes in generic metric theories of gravity, such as those described in \$3.1.4. This requires a general mathematical description of the shadow. In this direction, Ref. 3 developed a new general formalism to describe the shadow as an arbitrary polar curve expressed in terms of a Legendre expansion, which does not require any knowledge of the properties of the shadow (like its center or a primary shape), and allows one to introduce the various distortion parameters of the curve with respect to reference circles. These distortions can be implemented in a coordinate-independent manner while analysing the observational data. Moreover, this approach provides an accurate and robust method to measure the distortion of different parameters in the realistic case of a noisy shadow. In Fig. 8 we show a schematic picture that describes the distortions through various geometrical quantities. $\frac{3}{3}$

The idea behind this method is to develop a general description in terms of dimensionless parameters, translating the observations into a measure of the deviation from a given candidate theory of gravity, and subsequently defining confidence areas in the parameter space. This approach can be used in the analysis of mm-VLBI 

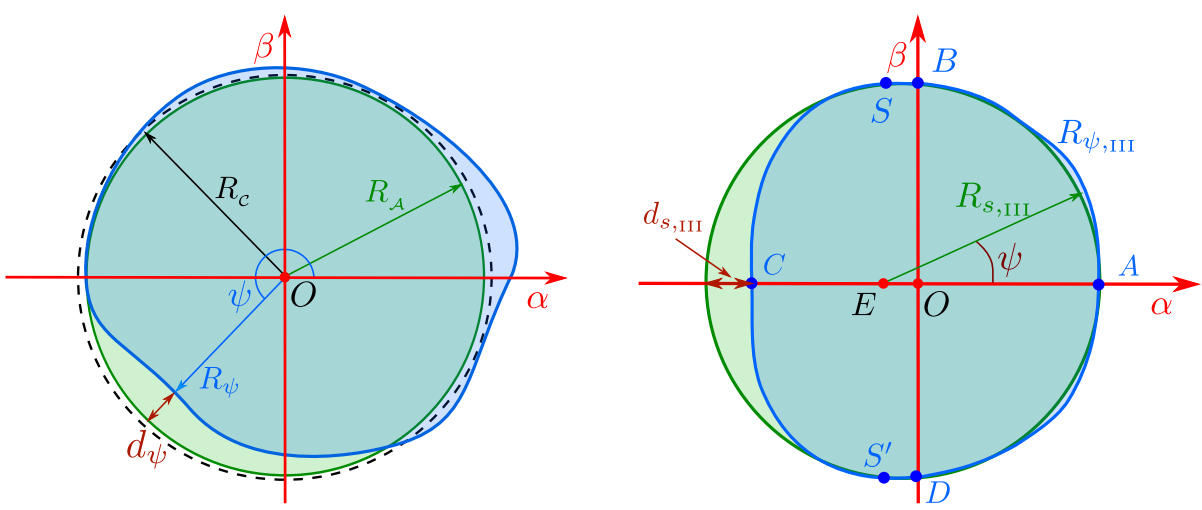

Fig. 8. Schematic representation of the distortion method adopted to study BH shadows by Ref. 3. The left panel shows the local distortion $d_{\psi}$ between the polar curve $R_{\psi}$ representing the black hole shadow (blue circle) and representative circles with circumference (dashed black) and area (green) radii, $R_{C}$ and $R_{A}$, respectively. The right panel shows the distortion parameter $d_{s \text {, III }}$ that measures the deviation between the Legendre expanded polar curve $R_{\psi, \text { III }}$ (blue circle) and the reference circle of radius $R_{s, \mathrm{II}}$ (green circle). The distortion is measured passing through the points $A, B, D$ and centered on point $E$. The zero-slope points are indicated with $S$ and $S^{\prime}$.

data $(3.1 .2$, to assess, in a quantitative manner, how accurately GR is confirmed by the observations. The next step is to build a generic numerical infrastructure able to produce the expected electromagnetic emission when the $\mathrm{BH}$ is considered in arbitrary metric theories of gravity. This computational platform may be coupled to GRMHD simulations and used to build a catalogue of $\mathrm{BH}$ images and emission properties in alternative theories of gravity. 171 The ultimate goal of $\mathrm{BH}$ shadow studies is to determine the theory of gravity that best describes the observations.

\subsection{Stellar orbits with near-infrared interferometry}

Monitoring of stellar orbits around Sgr A* enabled precise measurement of its mass (and distance), providing the clearest evidence for the existence of a SMBH at the center of our own Galaxy (see \$2.1.1. However, owing to the relatively large orbital distances of the currently known NIR stars around Sgr A* (a few thousand gravitational radii even for the tightest star S2; see Figure 11, there have been no dynamical measurements of its spin magnitude or orientation. In fact, relativistic effects that may enable the measurement of the $\mathrm{BH}$ spin are generally too small to be detected in the current experiments with single 8-m class telescopes. But these effects will come within reach by precisely measuring the orbits of stars with GRAVITY, a second-generation instrument on the Very Large Telescope Interferometer (VLTI), which is an adaptive-optics assisted optical interferometer. ${ }^{52}$ By providing astrometry with a precision of the order of $10 \mu$ as and imaging with a resolution of 4 mas, GRAVITY will push the sensitivity and accuracy of optical astrometry and interferometric imaging far beyond what is possible today. The first relativistic effect to be observed will be the peri-astron shift of the star S2 during its closest approach to the Galactic center SMBH in 2018. But in principle, stars 
with tighter orbits around Sgr A* (within a few hundred gravitational radii) can also be observed and their orbits determined precisely. Monitoring the precession of the orbits of these tighter stars and of their orbital planes will offer the possibility of measuring higher order relativistic effects as well. In particular the spin (and quadrupole moment) will cause a precession of the orbital plane of the star due to frame dragging, a phenomenon commonly referred to as Lense-Thirring precession $\frac{110}{}$ (Fig. 9). Since such a precession depends on two BH parameters, the spin and the quadrupole moment, measuring the Lense-Thirring precession for two (or more) stars, may allow us to disentangle their respective effects on the stellar orbits and therefore lead, in principle, to a test of the no-hair theorem. $\frac{166}{16}$
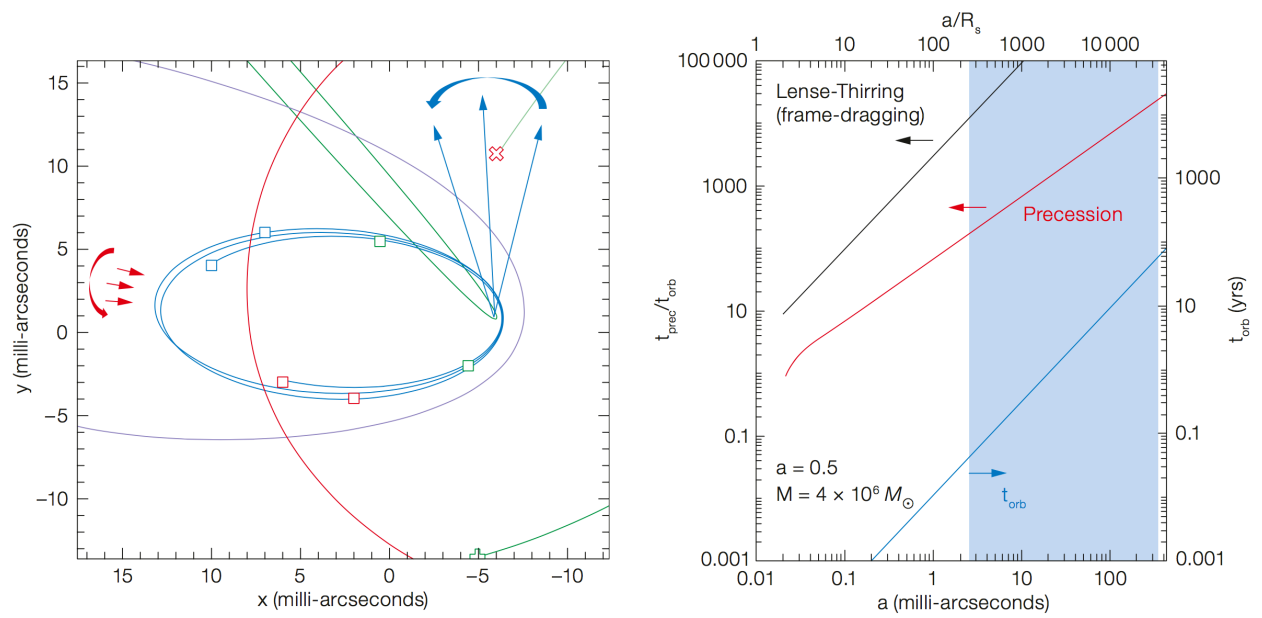

Fig. 9. Testing GR with stellar orbits. Stellar orbits (left panel) will be affected by the GR periastron shift (red arrows) and the Lense-Thirring precession of the orbital angular momentum (blue arrows). For small distances to the BH, the timescales of these relativistic effects are short enough (right panel) to be in reach of GRAVITY (blue shaded area). Adapted from Ref. 52

Besides the measurement of stellar orbits, an interesting prospect for GRAVITY will be the identification of the physical origin of periodic flares observed in the NIR and X-ray emission from $\mathrm{Sgr} \mathrm{A}^{*} .10169$ The $\sim$ hour-long timescale in the flare light curves provides a limit on the size scale of the emitting region, which corresponds to only a few $R_{\text {Sch }} \cdot{ }^{69}$ Three main explanations have been proposed for the origin of these flares: a jet with clumps of ejected material, ${ }^{\sqrt{6}}$ short-lived "hot spots" orbiting the $\mathrm{BH}, \underline{50}$ or statistical fluctuations in the accretion flow. $\stackrel{45}{ }$ Despite tremendous observing and modeling efforts, photometry and polarimetry alone have not been able to break the ambiguity between these scenarios. GRAVITY, by providing timeresolved astrometric measurements at the $10 \mu$ as level, will be finally able to settle the debate. ${ }^{[52}$ Interestingly, since GR effects dominate the detailed shape of the photo-center orbits, if the hot spot model turned out to be correct, the combination of time-resolved astrometry and photometry of a hot spot orbiting close to the ISCO may allow GRAVITY to directly probe the spacetime close to the event horizon, and eventually lead to an independent measurement of the $\mathrm{BH}$ spin and orientation. ${ }^{84}$ 


\subsection{Pulsars as probes of gravity}

So far the most precise tests of GR performed with strongly self-gravitating objects, as well as the most precise determinations of orbits outside the Solar system, have been achieved by pulsar timing. For instance, the emission of gravitational waves by a material system has been verified with pulsars to better than $0.1 \% .104$

Pulsars are rotating neutron stars that act like cosmic lighthouses, by emitting radio waves along their magnetic poles. For the observer on Earth, their emission appears as regular radio pulses in the sky, whose times of arrival at the radio telescope can be measured very precisely. When a pulsar is found in a binary system, it can be used as a probe of the binary spacetime, in a kind of clock-comparison experiment between the "pulsar-clock" and the hydrogen maser at the radio telescope. By this, relativistic effects in the proper time and the orbital motion of the pulsar (world-line of the pulsar) as well as propagation delays (null-geodesics of the pulsar signals) can be measured and compared to theoretical predictions. In GR but also within a wide class of alternative gravity theories, relativistic effects in binary pulsars can be modelled with the so-called "post-Keplerian" (PK) parameters. $.38 / 39141$ These PK parameters are theory-independent, phenomenological corrections to the Keplerian pulsar motion and the signal propagation, and describe, for instance, changes in the orientation and the period of the pulsar orbit, as well as additional delays in the pulses (like the Shapiro-delay), occurring as a result of the curvature of spacetime near the massive companion (see Ref. 114 for more details). Since these PK parameters are different, as functions of the Keplerian parameters and the component masses, in different theories of gravity, their measurement can be used to test GR and many of its alternatives. If the companion of the pulsar is a second neutron star, as for the Hulse-Taylor pulsar PSR B1913+16 $6^{86}$ and for the Double Pulsar PSR J0737-3039A/B, $\stackrel{301118}{1}$ timing observations of such compact binaries (with semi-major axes of about $1 R_{\odot}$ and orbital periods of only a few hours) can be used for precision tests of the interaction of two strongly self-gravitating bodies.107 On the other hand, if the companion of a pulsar in the binary is a white dwarf, the high asymmetry in compactness between pulsar and companion provides stringent tests for dipolar radiation, a prediction of many alternatives to GR.668

Besides pulsar binaries, some of the most stringent pulsar-based tests of GR and alternative theories are actually expected from a pulsar orbiting a BH. In such a case, we would not only expect the largest deviations from GR, at least for certain alternatives to GR, but we could also measure the $\mathrm{BH}$ properties, such as mass, spin and quadrupole moment, leading to a clean test of the no-hair theorem. Although pulsar-BH systems can provide unique benchmarks of theories of gravity, they are expected to be very rare and to date not a single pulsar-BH system has yet been found. In addition, since the effects related to the quadrupole moment scale with the third power of the BH mass, they are still extremely difficult to measure in the case of stellar mass BHs. ${ }^{[12]}$ A pulsar-SMBH binary, on the other hand, would be a perfect target for such tests. Luckily, the prospects of finding such a system 
can increase enormously near the Galactic center, where a large number of pulsars are expected to be orbiting Sgr A* (see 3.3 .1 ). Moreover, the enormous mass of Sgr A* would make the measurement of GR effects and deviations from GR a much

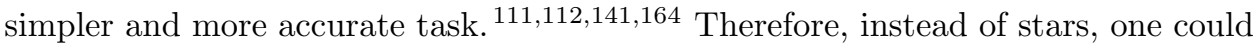
use pulsars along similarly tight orbits around Sgr $\mathrm{A}^{*}$ to probe its spacetime. In fact, it has been shown recently that, in order to perform a no-hair theorem test, the pulsar method might be much less affected by external perturbations, and even allow for wider orbits, than required for stars 144 (more below).

Pulsar timing has the power to provide accurate measurements of the mass, spin magnitude and 3D orientation, quadrupole moment, and distance of Sgr A*. There is a vast literature describing the methods to measure $\mathrm{BH}$-pulsar parameters via pulsar timing 106/111/112|144|164. Here we summarize the main concepts.

Ref. 114 describes how to measure accurate masses of binary pulsars from their PK parameters, and the same method can be applied to a pulsar orbiting Sgr A*. Since the pulsar is practically like a test particle, whose mass $\left(\sim 1.4 M_{\odot}\right)$ is mostly negligible with respect to the companion's mass $\left(\sim 4 \times 10^{6} M_{\odot}\right)$, the measurement of a single PK parameter allows one to determine the mass of Sgr A*, potentially with a precision of $\lesssim 10 M_{\odot}$ (corresponding to a relative precision of $<10^{-5}$ ), once a theory of gravity is assumed.111 At this point, the measurement of a second PK parameter already allows for a gravity test, since the inferred mass should agree with the one from the first PK parameter.111

Ref. 164 showed that in pulsar-BH binaries the Lense-Thirring precession allows one to measure the direction and magnitude of the $\mathrm{BH}$ spin. This can be achieved by measuring the rates of the secular precessions of the pulsar orbit (first and higherorder time derivatives) caused by the frame dragging. 1111 Ref. 144 demonstrated that, by combining the information of the proper motion of Sgr $A * 146$ with the orientation of the $\mathrm{BH}$ spin with respect to the pulsar orbit, it is possible to determine the $3 \mathrm{D}$ spin orientation. This would serve as an important input in the comparison with the image of the shadow of Sgr A* (\$3.1).

Once the mass and spin are measured, a Kerr spacetime is fully determined, and the measurement of any higher multipole moment provides a test of the no-hair theorem (sometimes also referred to as a test of the Kerr hypothesis). The quadrupole moment of Sgr $A^{*}$ leads to an additional secular precession of the pulsar orbit. However, this cannot be separated from the much larger secular Lense-Thirring precession. Luckily, the quadrupole moment also leads to a distinct periodic signal in the arrival times of the pulses, which allows for an independent extraction of the quadrupole moment. ${ }^{164}$ Based on mock data simulations, Ref. 111 demonstrated that for a pulsar with an orbital period of a few months it should be possible to determine the quadrupole moment of Sgr $\mathrm{A}^{*}$, solely from these periodic features in the timing residuals, with a precision of the order of $1 \%$, or even better, depending on the spin of Sgr A* and the eccentricity of the orbit.

pThe strength of the quadrupole effect scales with the square of the spin, and is therefore clearly 

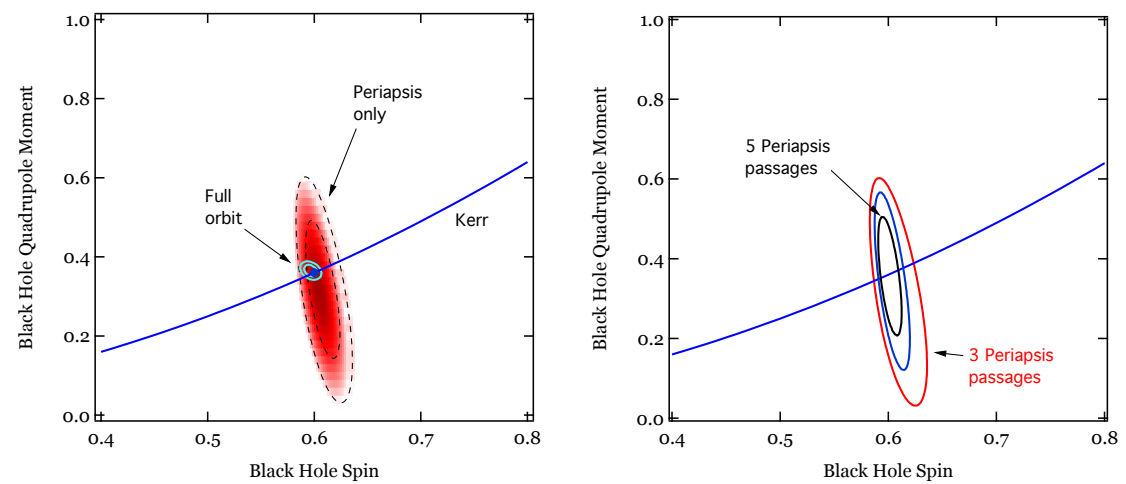

Fig. 10. The posterior likelihood of measuring the spin and quadrupole moment of SgrA* using pulsar timing. A Kerr $\mathrm{BH}$ is assumed with values of the spin of 0.6 and quadrupole moment of 0.36 ; the solid blue line shows the expected relation between the two parameters in the Kerr metric. The pulsar is assumed to have an orbital period of $0.5 \mathrm{yr}$ (orbital separation of $2400 \mathrm{R}_{g}$ ) and an eccentricity of 0.8 . The assumed timing uncertainty is $100 \mu \mathrm{s}$. (Left panel) Comparison between the uncertainties in the measurement when only three periapsis passages are considered (dashed curves showing the $68 \%$ and $95 \%$ confidence limits of the measurements) and those obtained with three full orbits (cyan curves). (Right panel) Improvement in the precision by increasing the number of periapsis passages from three (red curve) to five (black curve). Taken from Ref. 144.

The methods described above require that the motion of the pulsar around Sgr $A^{*}$ is mainly affected by the SMBH gravitational field, and external perturbations are negligible compared to the GR effects. As in the case of the S-stars, the pulsar orbit can experience external perturbations, for example from neighbouring stars or dark matter, which would lead to an additional precession of the orbit, which cannot be quantified a priori. $\frac{128 \mid 144}{}$ External perturbations are generally expected to be more prominent near apoapsis, when the gravitational effects from the SMBH are weaker and the pulsar motion is slower. On the other hand, in a highly eccentric orbit $(e \gtrsim 0.8)$ relativistic effects related to the gravitational field of the $\mathrm{BH}$ are most prominent around periapsis, where external effects are much more likely to be negligible. ${ }^{9163164}$ Consequently, there is considerable hope that even in the presence of external perturbations, relevant information on the SMBH parameters (mass, spin, and quadrupole moment) can still be extracted reliably, by taking only the small fraction of the orbit near the periapsis. In fact, Ref. 144 has demonstrated this in fully consistent mock data simulations. This is in striking contrast to stars, where we need at least two stars, which have to be monitored over several full orbits, in order to conduct a test of the Kerr hypothesis. 166

Fig. 10 shows the posterior likelihoods of measuring the spin and quadrupole moment of Sgr $\mathrm{A}^{*}$ with a pulsar for different observing runs assuming a timing precision of $100 \mu \mathrm{s}$, and a Kerr BH with a spin of 0.6. $\frac{1144}{1}$ Even in the case of a relatively low timing precision of $100 \mu \mathrm{s}$ a and the presence of external perturbations, the spin and quadrupole moment of Sgr A* can be measured with good precision by

less prominent for a slowly rotating $\mathrm{BH}$.

qRef. 144 considered also more optimistic scenarios, with timing precision of 1 and $10 \mu \mathrm{s}$. 
tracking the pulsar during a few periapsis passages, effectively demonstrating that a quantitative test of the no-hair theorem is possible after a few orbits.

In conclusion, detecting and timing a single normal pulsar in orbit around Sgr A* (similar to that of stars targeted by GRAVITY), and in the ideal case of negligible perturbations throughout the orbit, would allow one to measure the mass with a precision of a few to a few tens of $M_{\odot}$ (corresponding to a relative precision of $\lesssim 10^{-5}$ ), the spin to $0.1 \%$, and the quadrupole moment at a precision level of a few percent (or even better, depending on the size and orientation of the pulsar orbit and the spin of Sgr $\mathrm{A}^{*}$ ), thus providing a direct test of the no-hair theorem for a SMBH to an accuracy level of $1 \%$. This, in turn, may yield some of the most accurate tests of BHs in GR and in alternative theories and probe a completely new parameter space. But even in the presence of external perturbations, pulsar timing still has the potential to measure mass, spin and quadrupole moment of Sgr A* with good precision, by exploiting the characteristic timing residuals caused by the different relativistic effects during periapsis passages.

\subsubsection{Pulsars in the Galactic center}

Observational and theoretical considerations suggest the presence of a large number of neutron stars in the inner parsec of the Galaxy, with up to 100 normal pulsars and 1000 millisecond pulsar: ${ }^{\mathrm{T}}$ (e.g., see Refs. 35|167, and references therein).

Despite concentrated efforts to survey the central few tens of parsecs, $\frac{102105}{10}$ and the immediate vicinity ( $\lesssim 1 \mathrm{pc}$ ) of Sgr A* itself, $\underline{48 \mid 119}$ the number of pulsars discovered at the beginning of 2013 within $0.5^{\circ}$ of Sgr A* was only five. $\frac{40 \mid 98}{}$ This deficit was explained by severe interstellar scattering, which leads to temporal broadening of the pulses. This effect renders a pulsar essentially undetectable if the scattering time exceeds the pulse period; as was thought to be the case at typical search frequencies of around one to two GHz. Since the pulse scattering time scale, $\tau_{\mathrm{s}}$, is a strong function of radio observing frequency, $\nu$, where typically $\tau_{\mathrm{s}} \propto \nu^{-4}$, the strategy was therefore to conduct searches at increasing radio frequencies. The penalty associated with high frequency searches is, however, a severe drop in flux density due to the steep spectra of pulsars (average spectral index of -1.6).

The situation changed somewhat in April 2013, when radio emission from a transient magnetar was detected in the Galactic center. $\frac{49}{4}$ The source, now known as PSR J1745-2900, is located 2". 4 (or $0.1 \mathrm{pc)} \mathrm{from} \mathrm{Sgr} \mathrm{A*,22} \mathrm{which} \mathrm{is} \mathrm{within}$ the Bondi-Hoyle accretion radius. The angular scatter broadening of the source is consistent with that of $\left.\operatorname{Sgr} \mathrm{A}^{*}, 2122\right]$ while the rotation measure is by far the largest for any Galactic object (apart from Sgr A* itself). ${ }^{49}$ The dispersion measure is also the largest for any known pulsar, and the probability of a chance alignment with Sgr $\mathrm{A}^{*}$ is exceedingly small, $\stackrel{22149}{2}$ all together providing evidence for the proximity

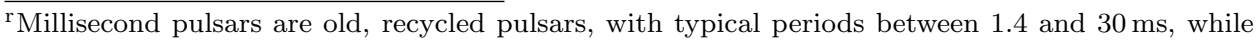
normal pulsars have average periods of 0.5 to $1 \mathrm{~s}$.
} 
of the magnetar (in 3D) to the Galactic center. Moreover, the proper motion of the pulsar is similar to the motion of massive stars orbiting Sgr A* in a clockwise disk. ${ }^{169}$ The predicted orbital period of PSR J1745-2900 is $\sim 700 \mathrm{yr}$, however the full 3D orbital motion around the central SMBH can be confirmed by measurements of acceleration in the proper motion. 22

Detecting the line-of-sight acceleration from pulsar timing measurements is unlikely because PSR J1745-2900 is a magnetar; a slowly-rotating pulsar (period $\sim 3.76 \mathrm{~s}$ ) with a strong magnetic field (in excess of $10^{14} \mathrm{G}$ ). Such objects cannot be used for precision timing experiments owing to their rotational instability and variable pulse profile shape. $\frac{31}{}$ Nevertheless, its detection suggests that a hidden pulsar population may be present. In fact, radio emitting magnetars are a rare type of neutron star ${ }^{5}$ with only three radio-loud magnetars previously known to exist in the Galaxy. Therefore the discovery of such an uncommon pulsar next to Sgr A* supports the hypothesis that many more ordinary radio pulsars should be present.

A surprising (but fortunate) implication of the magnetar discovery was that the pulse scatter broadening is effectively a factor of 1000 smaller than predicted: 154 in fact, with a pulse period of $3.76 \mathrm{~s}$, its radio emission should not be detectable at frequencies as low as $1.1 \mathrm{GHz}$, if hyper-strong scattering, as predicted in Refs. 36 37, were indeed present. A potential explanation is that the medium is highly turbulent (i.e., there is a lot of "weather"), resulting in a highly variable scattering, therefore the pulsars may be present but not detectable all the time. ${ }^{154}$ Another possibility is that the scattering towards the magnetar may not be representative of the entire Galactic center region, and stronger scattering could be present in other parts: i.e., the line-of-sight to Sgr A* could still be plagued with hyper-strong scattering as predicted in Refs. 36|37. Even if the latter is true, there are reasons to be optimistic for pulsar searches of the Galactic center at high radio frequencies because of the strong inverse frequency dependence of pulse scatter broadening (at high frequencies pulse scattering can be neglected ${ }^{114}$ ).

However, finding pulsars at such frequencies is intrinsically difficult, as their flux density decreases steeply with increasing frequency. So far, the number of pulsars detected at very high frequencies is rather small (nine at $32 \mathrm{GHz}$, four at $43 \mathrm{GHz}$ and one at $87 \mathrm{GHz}) \stackrel{113}{ }{ }^{113}$ PS J1745-2900 is an exception, owing to a very flat flux density spectrum, which has allowed its detection from a few $\mathrm{GHz}$ up to $225 \mathrm{GHz}$, which is the highest frequency at which a radio pulsar has been observed to date.159 The detection of pulsars at high frequencies has been mainly limited by the sensitivity of available mm-telescopes, but with the advent of next-generation mm-observatories, such as the LMT, phased-NOEMA, and phased-ALMA (see \$3.1.2), the hunt for pulsars around Sgr A* will enter a new phase. This next-generation instruments will provide sufficiently high sensitivity to allow the first systematic survey for pulsars at frequencies of about $90 \mathrm{GHz}$ (or higher) in the Galactic center. $\underline{61}$

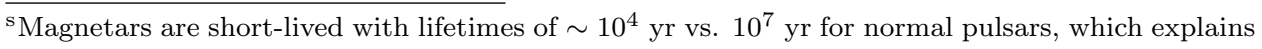
their rarity. 


\subsection{Combining the constraints from different techniques}

In previous sections, we have described the prospects of measuring the properties of the SMBH in the Galactic center and its spacetime using three types of observations: the BH shadow with the EHT, stars orbiting Sgr A* with GRAVITY, and pulsars with ALMA (and other telescopes). Although each type of observation is sensitive to (different) relativistic effects and may lead by itself to a measurement of the $\mathrm{BH}$ properties, it is only by combining the three techniques that it will be possible to assess systematics and quantify uncertainties in each measurement, leading to a precise, quantitative test of the validity of GR. There are a number of reasons.

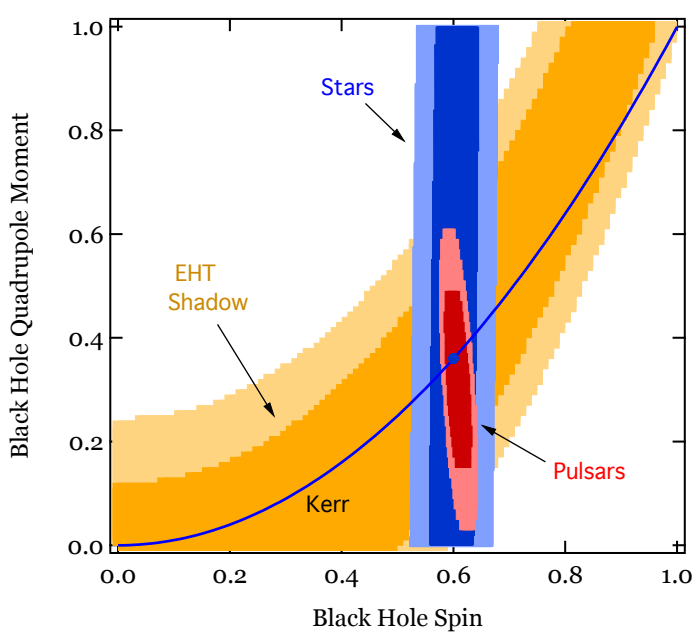

Fig. 11. Comparison of the posterior likelihood of measuring the spin and quadrupole moment of Sgr A* using the image of its shadow (gold), orbits of two stars (blue), and timing of three periapsis passages of a pulsar (red). The curves show the $68 \%$ (light colors) and 95\% (dark colors) confidence limits of the measurements. A Kerr BH is assumed with values of the spin of 0.6 and quadrupole moment of 0.36 (indicated by the blue dot). The solid blue line shows the expected relation between the two parameters in the Kerr metric. The combination of these three independent measurements can significantly increase our confidence in the estimate of the $\mathrm{BH}$ spin and quadrupole moment, thus providing a test of the no-hair theorem. Taken from Ref. 144.

Firstly, each measurement uses a very different observational technique (mmVLBI images of synchrotron emission, stellar astrometry with NIR interferometry, pulsar timing with radio telescopes) and is, therefore, affected by very different systematics, which can be more easily identified by comparing results from the three methods. Secondly, any difference in the measurements of the BH mass, spin, or quadrupole moment, from the three methods, can define the precision of these measurements. Thirdly, and most importantly, each type of observation is expected to lead to correlated uncertainties (or degeneracies) between the BH spin and quadrupole moment (e.g., see Figure 10 in the case of pulsars), as well as between the spin and potential deviations from the Kerr geometry (see e.g. Ref. 73). The combination of different methods can in fact break this degeneracy and therefore lead to independent estimates of the $\mathrm{BH}$ parameters and to a clean test of the Kerr metric. This has been demonstrated by Ref. 144, who show that the correlated uncertainties in the measurements of the spin and quadrupole moment using the orbits of stars and pulsars are along different directions in the parameter space to those obtained from measuring the shape and size of the shadow with 

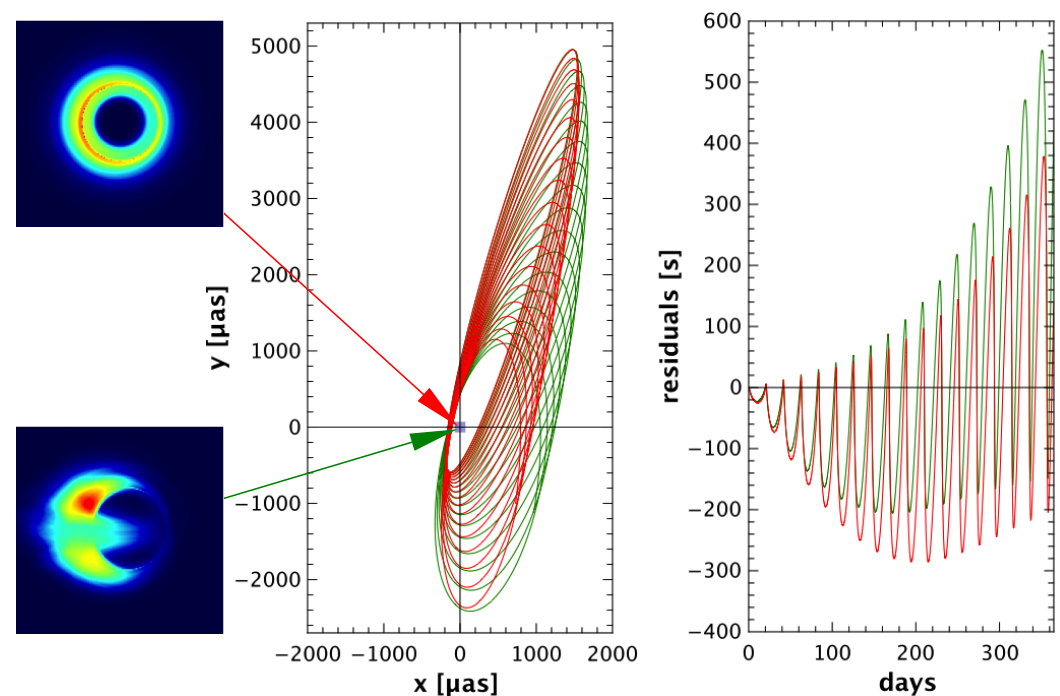

Fig. 12. GRMHD simulated images of Sgr $\mathrm{A}^{*}$ for two orientations (face-on and edge-on) of the spin axis (left) without instrumental effects, compared to potential pulsar orbits (middle) and timing signals (right) for these configurations (red indicating face-on, green indicating edge-on).

VLBI imaging. This is illustrated in Figure 11 which shows the Bayesian likelihood of simulated measurements of the spin and quadrupole moment for a Kerr BH (similar to the plot shown in Figure 10) with the three methods: EHT imaging of the BH shadow, GRAVITY observations of two stars, and timing observations of three periapsis passages of a pulsar ${ }^{144}$ Remarkably, the contours of the GRAVITY and pulsar-timing observations are nearly orthogonal to the contours of the EHT measurements, reducing the uncertainty of a combined measurement significantly.

It is interesting to note that while pulsars (and stars) probe the far-field (100s1000s $\left.R_{g}\right)$, the shadow image probes the near-field $\left(<10 \mathrm{~s} R_{g}\right)$. Both observations must nevertheless fit in the same model: it should be possible to predict the $\mathrm{BH}$ image from pulsar observations and then compare it with the VLBI measurements. This is illustrated in Figure 12, where we show two projected precessing pulsar orbits and the resulting timing residuals together with the expected VLBI images for two $\mathrm{BH}$ spin-orientations (face-on and edge-on, respectively). Both configurations have distinctive signatures in the image and in the timing, thereby over-constraining the model. Any difference between imaging, GR modeling, and pulsar timing will thus indicate the precision of the measurement of Sgr A*'s mass and spin. An independent third measurement could come from GRAVITY and eventually all three methods should intersect for a proper theory.

In fact, it could be the case that only the combination of the far-field measurements based on pulsars and stars, with the near field tests from imaging, has the power to reveal a deviation from Kerr. This has been the subject of a recent study by Ref. 73, who showed that the near-field image by itself might not be able to detect a deviation from Kerr (as an illustration, see their Figure 5a). However, once 
the spin measurement from the pulsar done in the Kerr-like far field is combined with the constraints from modeling the shadow, one can recover the deformation (in their case, parameter $\epsilon_{3}^{\mathrm{t}}$ ), and test for a violation of the Kerr hypothesis.

In summary, although the measurement of spacetime around a $\mathrm{BH}$ from each type of observation will be ground-breaking in itself, it is only the cross-comparison of the predictions from different methods that has the power to provide a fundamental test of GR, and therefore lead to a true breakthrough.

\section{Summary and Conclusions}

GR has just turned 100 years old, and yet no other theory of gravity is equally successful at describing the complex phenomenology that astronomical and cosmological observations provide, both on the smallest scales of the Solar System and on the largest cosmological scales. In fact, GR has successfully passed all tests carried out both in the weak field limit (as in the Solar System) and for strongly self-gravitating bodies in pulsar binary systems. While these tests have confirmed GR as the standard theory of gravity, tests in the strong field regime are still missing. The strongest gravitational fields are expected to be around BHs, especially SMBHs. Therefore the most promising tests of GR are those aiming to probe the spacetime around SMBHs, where the largest deviations from GR are expected and/or alternative theories of gravity may apply.

While there are many $\mathrm{BH}$ candidates in the universe, the most compelling evidence for the existence of a SMBH is provided by the radio source Sgr $\mathrm{A}^{*}$ in the center of our own Galaxy. With its large mass of $4.3 \pm 0.4 \times 10^{6} M_{\odot}$ and at a distance of only $8.34 \pm 0.15 \mathrm{kpc}$, Sgr $\mathrm{A}^{*}$ is the prime target for $\mathrm{BH}$ and GR experimental studies.

The main goal of BlackHoleCam is to conduct GR tests in a strong-field regime that has not been explored directly so far, using three different types of observations of Sgr A* across the electromagnetic spectrum with new-generation instruments.

The first experiment consists of making a standard astronomical image of the accretion flow around Sgr A*. At its center, GR predicts the appearance of a $\mathrm{BH}$ "shadow", which is a gravitationally lensed image of the photon capture sphere and has a diameter of about $5 \mathrm{R}_{\mathrm{Sch}} \sim 50 \mu \mathrm{as}$ (as seen from Earth). The plasma accreting onto the $\mathrm{SMBH}$ radiates synchrotron emission that peaks at (sub)mm waves and it is optically thin, thus mm-VLBI observations can enable us to see the innermost reaches of an event horizon. The EHT, a virtually Earth-sized telescope which uses the mm-VLBI technique, is being assembled at the moment, and will soon achieve the resolving power to finally resolve horizon scales and make an image of the shadow cast by the SMBH at the Galactic center. This will not only provide convincing evidence about the existence of an event horizon (and therefore of $\mathrm{BHs}$ ), but since the size and the shape of the shadow depend primarily on the underlying spacetime (besides the basic BH parameters - see below), it will also provide a first-order test of the validity of GR and/or alternative theories of gravity (which 
also predict BHs and shadows). In order to carry out such a strong-field test, in BlackHoleCam we are building an appropriate theoretical framework to model both the spacetime in generic theories of gravity as well as the emission and dynamics of the plasma near the BH with GRMHD simulations. By comparing shadow images from EHT observations with model predictions, we aim to measure deviations from GR and thus test it against alternative theories of gravity in the strong field cases.

While making the first image of a $\mathrm{BH}$ will be a breakthrough discovery, it will not be sufficient by itself to provide a precision test of GR. In fact, the shadow's properties depend on both the BH parameters and its spacetime, resulting in an inherent degeneracy between e.g. the $\mathrm{BH}$ spin and the deviation parameters of a given Kerr-like metric. It then becomes key to reduce the free parameters by determining the $\mathrm{BH}$ parameters (mass, spin, inclination) independently from the imaging. We plan to do this by monitoring stellar orbits with the forthcoming NIR interferometer at the VLT, GRAVITY, which can detect orbital precessions induced by relativistic effects like the frame-dragging, enabling a measurement of the spin of Sgr A*. Since the uncertainty on the latter is correlated with that of the quadrupole moment, a further independent measurement is required to break the degeneracy.

The third method is provided by radio observations of pulsars, which are thought to populate the Galactic center. By timing a pulsar on a tight orbit (period $<1$ year) around Sgr A*, we may detect distinctive signatures of a number of relativistic and precessional effects, potentially allowing us to determine the BH's mass to one part in a million, its spin to tenths of a percent, and the quadrupole moment to a few percent, respectively. The recent detection of a magnetar at $0.1 \mathrm{pc}$ from Sgr $\mathrm{A}^{*}$ has renewed hopes of finding a pulsar in tight orbit around Sgr $\mathrm{A}^{*}$, and future surveys at high frequencies with ALMA hold the promise to achieve that.

A last point worth stressing is that since the observables of the experiments described here are very different and are therefore subject to different systematics, the combination of three independent measurements would provide a very convincing case, resulting either in an increase in our confidence in the validity of GR in the strong-field regime, or in very serious consequences for the foundations of the theory. Ultimately, such experiments should help us assess which theory of gravity best describes the astrophysical observations, and thus the observable Universe.

In conclusion, the combination of event-horizon imaging and $\mathrm{BH}$ modeling, along with pulsar timing and stellar dynamics, can now transform the Galactic center into a precision-astrophysics and fundamental-physics laboratory for testing GR in its most extreme limits, allowing us to explore the fine structure of the fabric of spacetime in any metric theory of gravity.

\section{Acknowledgments}

This work is supported by the ERC Synergy Grant "BlackHoleCam: Imaging the Event Horizon of Black Holes" (Grant 610058). 


\section{References}

1. Abbott, B. P., et al. 2016, Phy. Rev. Lett., 116, 061102

2. Abdujabbarov, A., Atamurotov, F., et al. 2013, Ap\&SS, 344, 429

3. Abdujabbarov, A. A., Rezzolla, L., \& Ahmedov, B. J. 2015, MNRAS, 454, 2423

4. Amarilla, L., Eiroa, E. F., \& Giribet, G. 2010, Phy. Rev. D, 81, 124045

5. Amarilla, L., \& Eiroa, E. F. 2013, Phy. Rev. D, 87, 044057

6. Antoniadis, J., Freire, P. C. C., Wex, N., et al. 2013 Science, 340, 448

7. Atamurotov, F., Abdujabbarov, A., Ahmedov, B. 2013, Phy. Rev. D, 88, 064004

8. Atamurotov, F., Ghosh, S. G., \& Ahmedov, B. 2015, arXiv:1506.03690

9. Angélil, R. \& Saha, P. 2014, MNRAS, 444, 3780

10. Baganoff, F. K., Bautz, M. W., Brandt, W. N., et al. 2001, Nature, 413, 45

11. Balick, B., \& Brown, R. L. 1974, ApJ, 194, 265

12. Balbus, S. A., \& Hawley, J. F. 1998, Rev. Mod. Phys., 70, 1

13. Bambi, C. \& Freese, K. 2009, Phy. Rev. D, 79, 043002

14. Bambi, C., \& Yoshida, N. 2010, Class. Quantum Grav., 27, 205006

15. Bardeen, J. M. 1973, in Black Holes, ed. C. DeWitt \& B.S. DeWitt, pages 215-239 (New York: Gordon \& Breach)

16. Blandford, R. D., \& Begelman, M. C. 1999, MNRAS, 303, L1

17. Blecher, T. D., Deane, R. P., Bernardi, G., \& Smirnov, O. M. 2016, MNRAS, submitted

18. Bower, G. C., Falcke, H., Herrnstein, R. M., et al. 2004, Science, 304, 704

19. Bower, G. C., Goss, W. M., Falcke, H., et al. 2006, ApJL, 648, L127

20. Bower, G. C., Markoff, S., Brunthaler, A., et al. 2014a, ApJ, 790, 1

21. Bower, G. C., Deller, A., Demorest, P., et al. 2014b, ApJL, 780, L2

22. Bower, G. C., Deller, A., Demorest, P., et al. 2015, ApJL, 798, L2

23. Bower, G. C., Wright, M.C.H., Falcke, H., \& Backer, D. C. 2003, ApJ, 588, 331

24. Brinkerink, C. D., Falcke, H., Law, C. J., et al. 2015, A\&A, 576, A41

25. Brinkerink, C. D., et al. 2016, submitted to $M N R A S$

26. Broderick, A. E., Fish, V. L., Doeleman, S. S., \& Loeb, A. 2009, ApJ, 697, 45

27. Broderick, A. E., Fish, V. L., Doeleman, S. S., \& Loeb, A. 2011, ApJ, 735, 110

28. Broderick, A. E., Johannsen, T., Loeb, A., \& Psaltis, D. 2014, ApJ, 784, 7

29. Broderick, A. E. \& Loeb, A. 2006, MNRAS, 367, 905

30. Burgay, M., D'Amico, N., Possenti, A., et al. 2003, Nature, 426, 531

31. Camilo, F., Cognard, I., Ransom, S. M., et al. 2007, ApJ, 663, 497

32. Capozziello, S. \& De Laurentis, M. 2011, Phys.Rept. 509, 167

33. V. Cardoso, P. Pani and J. Rico, 2014, Phys. Rev. D, 89, 064007

34. Chatzopoulos, S., Fritz, T. K., Gerhard, O., et al. 2015, MNRAS, 447, 948

35. Chennamangalam, J. \& Lorimer, D.R. 2014, MNRAS, 440, L86

36. Cordes, J. M. \& Lazio T. J. W. 2001, ApJL, 562, L157

37. Cordes, J. M. \& Lazio T. J. W. 2002, arXiv:astro-ph/0207156

38. Damour, T. \& Deruelle, N. 1986, Ann. Inst. H. Poincaré, 44, 263

39. Damour, T. \& Taylor, J. H. 1992, Phy. Rev. D, 45, 1840 
40. Deneva, J. S., Cordes, J. M., Lazio, T. J. W. 2009, ApJL, 702, L177

41. De Laurentis, M., \& De Martino, I. 2013, MNRAS, 431, 741

42. de Vries, A. 2000, Class. Quantum Grav., 17, 123

43. De Villiers, J.-P., Hawley, J. F., \& Krolik, J. H. 2003, ApJ, 599, 1238

44. Dexter, J., Agol, E., Fragile, P. C., \& McKinney, J. C. 2010, ApJ, 717, 1092

45. Do, T., Ghez, A. M., Morris, M. R., et al. 2009, ApJ, 691, 1021

46. Doeleman, S. S., Weintroub, J., Rogers, A. E. E., et al. 2008, Nature, 455, 78

47. Doeleman, S. S., Fish, V. L., Broderick, A. E., et al. 2009, ApJ, 695, 59

48. Eatough, R. P., Kramer, M., Klein, B., et al. 2013a, in IAU Symposium 291, ed. van Leeuwen, CUP, 291, 382

49. Eatough, R. P., Falcke, H., Karuppusamy, R., et al. 2013b, Nature, 501, 391

50. Eckart, A., Baganoff, F. K., Schödel, R., et al. 2006, A\&\&A, 450, 535

51. Dyson, F. W. and Eddington, A. S. and C., Davidson 1920, Phil. Trans. R. Soc. Lond., A 220, 291

52. Eisenhauer, F., Perrin, G., Brandner, W., et al. 2011, The Messenger, 143, 16

53. Falcke, H., Goss, W. M., Matsuo, H., et al. 1998, ApJ, 499, 731

54. Falcke, H., Markoff, S., \& Bower, G. C. 2009, A\& $A$, 496, 77

55. Falcke, H., Mannheim, K., \& Biermann, P. L. 1993, A\& $A$, 278, L1

56. Falcke, H., \& Markoff, S. 2000, A\& A, 362, 113

57. Falcke, H., \& Markoff, S. B. 2013, Class. Quantum Grav., 30, 244003

58. Falcke, H., Melia, F. \& Agol, E. 2000, ApJL, 528, L13

59. Fish, V. L., Broderick, A. E, Doeleman, S.S, \& Loeb, A. 2009a, ApJL, 692, L14

60. Fish, V. L., Doeleman, S. S., Beaudoin, C., et al. 2011, ApJL, 727, L36

61. Fish, V., Alef, W., Anderson, J., et al. 2013, arXiv:1309.3519

62. Fish, V. L., Johnson, M. D., Lu, R.-S., et al. 2014, ApJ, 795, 134

63. Fish, V. L., Johnson, M. D., Doeleman, S. S., et al. 2016, ApJ, 820, 90

64. Fishbone, L. G., \& Moncrief, V. 1976, ApJ, 207, 962

65. Font, J. A. \& Daigne, F. 2002, ApJ, 581, L23

66. Foucart, F., Chandra, M., Gammie, C., Quataert, E. 2016, MNRAS, 456, 1332

67. Fraga-Encinas, R., Mościbrodzka, et al. 2016, Aछ A, 588, A57

68. Freire, P. C. C., Wex, N., Esposito-Farèse, G., et al. 2012, MNRAS, 423, 3328

69. Genzel, R., Schödel, R., Ott, T., et al. 2003, Nature, 425, 934

70. Genzel, R., Eisenhauer, F., \& Gillessen, S. 2010, Rev. Mod. Phys., 82, 3121

71. Genzel, R., Pichon, C., Eckart, A., et al. 2000, MNRAS, 317, 348

72. Genzel, R., \& Townes, C. H. 1987, A\&3AR, 25, 377

73. Ghasemi-Nodehi, M., Li, Z., \& Bambi, C. 2015, Eur. Phys. J. C, 75, 315

74. Ghez, A. M., Morris, M., Becklin, E. E., et al. 2000, Nature, 407, 349

75. Ghez, A. M., Salim, S., Weinberg, N. N., et al. 2008, ApJ, 689, 1044

76. Gillessen, S., Eisenhauer, F., Trippe, S., et al. 2009a, ApJ, 692, 1075

77. Gillessen, S., Eisenhauer, F., Fritz, T. K., et al. 2009b, ApJL, 707, L114

78. Gillessen, S., Eisenhauer, F., Fritz, T. K., et al. 2013, Advancing the Physics of Cosmic Distances, 289, 29 
79. Grenzebach, A., Perlick, V., Lammerzahl, C. 2014, Phy. Rev. D, 89, 124004

80. Grenzebach, A., Perlick, V., Lammerzahl, C. 2015, Int. J. Mod. Phys. D, 24, 1542024

81. Grenzebach, A., The Shadow of Black Holes - An Analytic Description, SpringerBriefs in Physics, Springer, Heidelberg (2016).

82. Greve, A., Torres, M., Wink, J. E., et al. 1995, A $6 A$, 299, L33

83. Hamaker, J. P., Bregman, J. D., \& Sault, R. J. 1996, A $\mathscr{E} A S, 117,137$

84. Hamaus, N., Paumard, T., Müller, T., et al. 2009, ApJ, 692, 902

85. Hawking, S. W. 1972, Commun. Math. Phys., 25, 152

86. Hulse, R. A., \& Taylor, J. H. 1975, ApJL, 195, L51

87. Israel, W. 1967, Physical Review, 164, 1776

88. Johannsen, T. 2013, Phy. Rev. D, 88, 044002

89. Johannsen, T., Broderick, A. E., et al. 2016, Phy. Rev. Lett., 116, 031101

90. Johannsen, T. 2016, Class. Quantum Grav., 33, 113001

91. Johannsen, T. 2016, Class. Quantum Grav., 33, 124001

92. Johannsen, T., \& Psaltis, D. 2010a, ApJ, 716, 187

93. Johannsen, T., \& Psaltis, D. 2010b, ApJ, 718, 446

94. Johannsen, T., \& Psaltis, D. 2011, Phy. Rev. D, 83, 124015

95. Johnson, M. D., Fish, V. L., Doeleman, S. S., et al. 2014, ApJ, 794, 150

96. Johnson, M. D., Fish, V. L., Doeleman, S. S., et al. 2015, Science, 350, 1242

97. Johnson, M. D., Kovalev, Y. Y., Gwinn, C. R., et al. 2016, ApJL, 820, L10

98. Johnston, S., Kramer, M., Lorimer, D.R., et al. 2006, MNRAS, 373, L6

99. Jones, R. C. 1941, Journal of the Optical Society of America, 31, 488

100. P. Kanti and R. A. Konoplya, 2006, Phy. Rev. D, 73, 044002

101. Keppens, R., Meliani, Z., van Marle, A. J., et al. 2012, J.Co.Ph., 231, 718

102. Klein, B., Kramer, M., Müller, P., et al. 2004, IAU Symp., 218, 133

103. Konoplya, R., Rezzolla, L., \& Zhidenko, A. 2016, Phy. Rev. D, 93, 064015

104. Kramer, M., Probing gravitation with pulsars, IAU, 8:19-26, 2012

105. Kramer, M., et al. 2000, in "IAU Colloq. 177: Pulsar Astronomy - 2000 and Beyond", eds. M. Kramer, N. Wex, \& R. Wielebinski, ASPC, 202, 37

106. Kramer, M., Backer, D. C., Cordes, J. M., et al. 2004, NewAR, 48, 993

107. Kramer, M., Stairs, I. H., Manchester, R. N., et al. 2006, Science, 314, 97

108. Krichbaum, T. P., Zensus, J. A., Witzel, A., et al. 1993, A\&A, 274, L37

109. Krichbaum, T. P., Graham, D. A., Witzel, A., et al. 1998, A\&A, 335, L106

110. Lense, J., \& Thirring, H. 1918, Physikalische Zeitschrift, 19,

111. Liu, K., Wex, N., Kramer, M., Cordes, J. M., Lazio, T. J. 2012, ApJ, 747, 1

112. Liu, K., Eatough, R. P., Wex, N., \& Kramer, M. 2014, MNRAS, 445, 3115

113. Löhmer, O., Jessner, A., Kramer, M., et al. 2008, A\&A, 480, 623

114. Lorimer, D. R. \& Kramer, M. 2004, Handbook of Pulsar Astronomy, Cambridge University Press, Cambridge

115. Lu, R.-S., Krichbaum, T. P., Eckart, A., et al. 2011, A\&A, 525, A76

116. Lu, R.-S., Roelofs, F., Fish, V. L., et al. 2016, ApJ, 817, 173 
117. Luminet, J.-P. 1979, A\&A, 75, 228

118. Lyne, A. G., Burgay, M., Kramer, M., et al. 2004, Science, 303, 1153

119. Macquart, J.-P., Kanekar, N., Frail, D. A. \& Ransom S. 2010, ApJ, 715, 939

120. Mahadevan, R., \& Quataert, E. 1997, ApJ, 490, 605

121. Markoff, S., Bower, G. C., \& Falcke, H. 2007, MNRAS, 379, 1519

122. Marrone, D. P., Moran, J. M., Zhao, J.-H., \& Rao, R. 2006, ApJ, 640, 308

123. Marrone, D. P., Moran, J. M., Zhao, J.-H., \& Rao, R. 2007, ApJL, 654, L57

124. McKinney, J. C. 2006, MNRAS, 368, 1561

125. McKinney, J. C., Tchekovskoy, A., \& Blandford, R. 2012, MNRAS, 423, 3083

126. Melia, F., \& Falcke, H. 2001, A\&AR, 39, 309

127. Meliani, Z., Mizuno, Y., Olivares, H., Porth, O., Rezzolla, L., \& Younsi, Z. 2016, $A \mathscr{E} A$, in preparation

128. Merritt, D., Alexander, T., et al. 2010, Phy. Rev. D, 81, 062002

129. Meyer, L., Ghez, A. M., Schödel, R., et al. 2012, Science, 338, 84

130. Mościbrodzka, M., Gammie, C. F., Dolence, J. C., et al. 2009, ApJ, 706, 497

131. Mościbrodzka, M., \& Falcke, H. 2013, A $6 A$, 559, L3

132. Mościbrodzka, M., Falcke, H., et al. 2014, $A \mathscr{\mho} A$, 570, A7

133. Nagar, N. M., Falcke, H., \& Wilson, A. S. 2005, A\&A A, 435, 521

134. Narayan, R. 1992, Philosophical Transactions of the Royal Society of London Series A, 341, 151

135. Narayan, R. \& Yi, I. 1995, ApJ, 452, 710

136. Narayan, R., Mahadevan, R., Grindlay, J. E., et al. 1998, ApJ, 492, 554

137. Newman, E. T., \& Janis, A. I. 1965, Journal of Mathematical Physics, 6, 915

138. Noordam, J. E., \& Smirnov, O. M. 2010, A\&A, 524, A61

139. Ortiz-León, G. N., Johnson, M. D., Doeleman, S. S., et al. 2016, ApJ, 824, 40

140. Perlick, V. 2004, Living Reviews in Relativity, 7,

141. Pfahl, E. \& Loeb, A. 2004, ApJ, 615, 253

142. Porth, O., Xia, C., Hendrix, T., Moschou, S., Keppens, R. 2014, ApJS, 214, 4

143. Porth, O. et al. 2016, in preparation

144. Psaltis, D., Wex, N., \& Kramer, M. 2016, ApJ, 818, 121

145. Rauch, C., Ros, E., Krichbaum, T. P., et al. 2016, A\&A, 587, A37

146. Reid, M. J. \& Brunthaler, A., 2004, ApJ, 616, 872

147. Reid, M. J., Menten, K. M., Brunthaler, A., et al. 2014, ApJ, 783, 130

148. Rezzolla, L., \& Zhidenko, A. 2014, Phy. Rev. D, 90, 084009

149. Ricarte, A., \& Dexter, J. 2015, MNRAS, 446, 1973

150. Robinson, D. C. 1975, Phy. Rev. Lett., 34, 905

151. Sen, A. 1992, Phy. Rev. D, 69, 1006

152. Schödel, R., Ott, T., Genzel, R., et al. 2002, Nature, 419, 694

153. Shcherbakov, R. V., Penna, R. F., \& McKinney, J. C. 2012, ApJ, 755, 133

154. Spitler, L. G., Lee, K. J., Eatough, R. P., et al., 2014, ApJL, 780, L3

155. Synge, J. L. 1966, MNRAS, 131, 463

156. Tchekovskoy, A., Narayan, R., \& McKinney, J. C. 2011, MNRAS, 418, L79 
157. Thompson, A. R., Moran, J. M., \& Swenson, G. W. 2007, Interferometry and Synthesis in Radio Astronomy, John Wiley Sons, 2007.

158. Tilanus, R. P. J., Krichbaum, T. P., Zensus, J. A., et al. 2014, arXiv:1406.4650

159. Torne, P., Eatough, R. P., Karuppusamy, R., et al. 2015, MNRAS, 451, L50

160. van Langevelde, H. J., Frail, D. A., et al. 1992, ApJ, 396, 686

161. Vigeland, S., Yunes, N., \& Stein, L. C. 2011, Phy. Rev. D, 83, 104027

162. Wagner, J., Roy, A. L., Krichbaum, T. P., et al. 2015, A\&4A, 581, A32

163. Wex, N. 1995, Class. Quantum Grav., 12, 983

164. Wex, N. \& Kopeikin, S. 1999, ApJ, 513, 388

165. Will, C. M. 2005, Phy. Rev. D, 71, 084027

166. Will, C. M. 2008, ApJL, 674, L25

167. Wharton R. S., Chatterjee, S., Cordes, J. M., et al. 2012, ApJ, 753, 108

168. Yagi, K., Stein, L. C., \& Yunes, N. 2016, Phy. Rev. D, 93, 024010

169. Yelda, S., Ghez, A. M., Lu, J. R., et al. 2014, ApJ, 783, 131

170. Young, P. J. 1976, Phy. Rev. D, 14, 3281

171. Younsi, Z., Zhidenko, A., Rezzolla, L., Konoplya, K., \& Mizuno, Y. 2016, in preparation

172. Yuan, F., \& Narayan, R. 2014, ARAA, 52, 529

173. Yuan, F., Quataert, E., \& Narayan, R. 2003, ApJ, 598, 301

174. Yuan, F., Markoff, S., \& Falcke, H. 2002, AछA, 383, 854

175. Yuan, F., Wu, M., \& Bu, D. 2012, ApJ, 761, 129

176. Yuan, F., Bu, D., \& Wu, M. 2012, ApJ, 761, 130

177. Yumoto, A., Nitta, D., et al. 2012, Phy. Rev. D, 86, 103001 